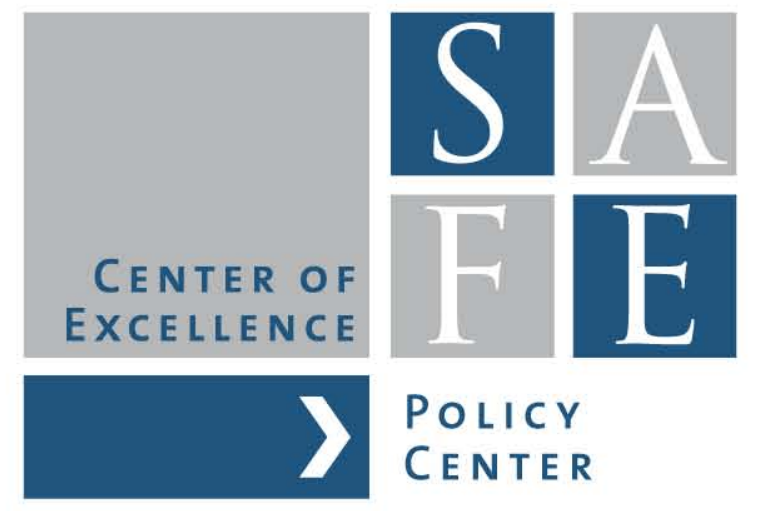

Peter Gomber und Benedikt Jäger

\title{
MiFID: Eine systematische Analyse der Zielerreichung
}

White Paper Series No. 14

Center of Excellence SAFE Sustainable Architecture for Finance in Europe A cooperation of the Center for Financial Studies and Goethe University Frankfurt 
This paper was also published in: Zeitschrift fuer Bankrecht und Bankwirtschaft, 2014 No. 1, pp. 4053; 2014 of the Center of Excellence SAFE or its staff. 


\title{
MiFID: Eine systematische Analyse der Zielerreichung
}

\author{
Peter Gomber, Benedikt Jäger ${ }^{1}$
}

Mai 2014

Inhaltsverzeichnis



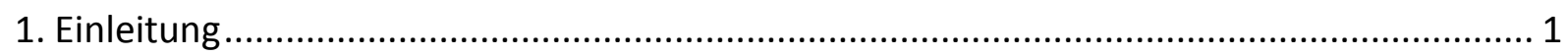

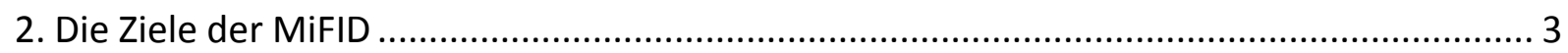

2.1. Identifizierung der MiFID-Ziele ......................................................................... 3

2.2. Die MiFID-Ziele: Effizienz und Kostensenkung ................................................ 4

2.3. Die MiFID-Zwischenziele: Wettbewerb, Integration, Transparenz und Integrität.. 6



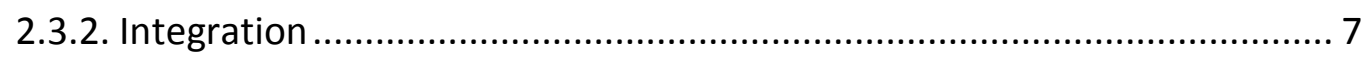

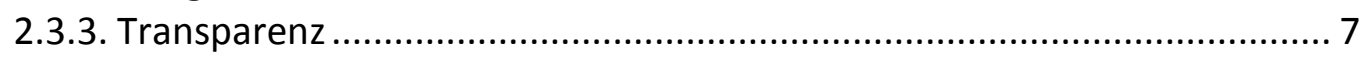



2.4. Die Wirkung der MiFID-Zwischenziele auf die MiFID-Ziele .................................. 8

3. Die Instrumente zur MiFID-Zielerreichung.................................................................. 10

3.1. Wettbewerb über die Aufhebung der Konzentrationsregel ............................... 10

3.2. Integration der fragmentierten Liquidität und Handelsinformationen ................ 11

3.3. Transparenz über die Offenlegung der Liquidität der Handelsplätze .................. 12

3.4. Integrität über das Best Execution Konzept ......................................................... 13

4. Die Bewertung der MiFID-Zielerreichung.................................................................... 13

4.1. Systematischer Review und Metaanalyse ....................................................... 13

4.2. Bewertung der MiFID-Zielerreichung anhand der wissenschaftlichen Literatur.. 18



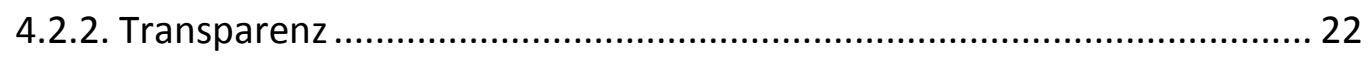

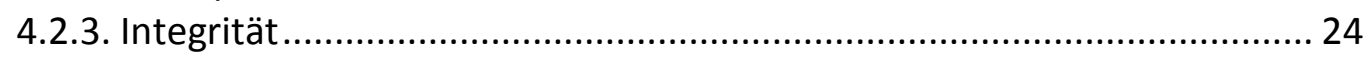

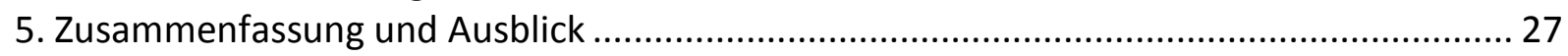

\section{Zusammenfassung}

Der vorliegende Artikel analysiert systematisch die Erreichung der MiFID-Ziele anhand der wissenschaftlichen Literatur. Ziel der MiFID ist es, die Rahmenbedingungen für einen effizienten und kostengünstigen Wertpapierhandel zu schaffen. Erreicht werden soll dies durch die Verschärfung des Wettbewerbs, die Integration der Märkte, die Offenlegung von Handelsintentionen und -geschäften sowie die Stärkung der rechtlichen Position der Investoren. Im Ergebnis zeigt sich, dass die Förderung des Wettbewerbes als erfolgreich bewertet wird, aber die regulatorischen Möglichkeiten der Marktintegration nicht ausgeschöpft werden. Ferner wird die Forderung nach einheitlichen Transparenzbestimmungen für alle Ordermechanismen nur teilweise umgesetzt. Der Anleger erfährt letztlich gegenüber Finanzintermediären durch die MiFID keinen höheren Schutz.

\footnotetext{
${ }^{1}$ Prof. Dr. Peter Gomber, M. Sc. Benedikt Jäger, Professur für e-Finance, Universität Frankfurt
} 


\section{Einleitung}

Die Legitimation von Regulierung stellt in der Ökonomie einen kontinuierlichen Diskurs dar. ${ }^{2}$ Während einige Wirtschaftswissenschaftler jede Form öffentlicher Steuerung ablehnen ${ }^{3}$, sprechen sich andere für massive Staatsinterventionen im Finanzsektor aus. ${ }^{4}$ Bezogen auf Wertpapiermärkte besteht zudem in empirischen Untersuchungen Uneinigkeit, ob stark regulierte Handelsplätze in ihrer Leistungsfähigkeit gering regulierte Handelsplätze übertreffen. ${ }^{5}$ Ist die Entscheidung zugunsten staatlicher Einflussnahme in den Wertpapierhandel gefallen, gilt die Kombination aus Regulierung ex ante verbunden mit privatrechtlicher Durchsetzung ex post als optimal. ${ }^{6}$

Entwicklungen in der wirtschaftlichen Integration, wie die Einführung des freien Kapitalverkehrs oder die Währungsunion, und in der Technologie, wie der computergestützte Börsenhandel, ergaben die Notwendigkeit, die unterschiedlichem nationalen Recht unterliegenden europäischen Finanzmärkte zu harmonisieren. ${ }^{7}$ Ausgangspunkt zur Integration der europäischen Kapitalmärkte ist der Financial Services Action Plan (FSAP), den die Europäische Kommission im Mai 1999 vorlegte. Aus diesem übergeordneten finanzpolitischen Plan wurden gesetzgeberische Maßnahmen abgeleitet, die die Form einer Regulierung ${ }^{8}$ oder einer Richtlinie (Direktive) ${ }^{9}$ annehmen können. Zentraler Eckpfeiler des FSAP ist die am 30. April 2004 verabschiedete und ab 1. November 2007 durch Geregelte Märkte und Wertpapierdienstleistungsunternehmen anzuwendende Richtlinie über Märkte für Finanzinstrumente (im Fol-

\footnotetext{
${ }^{2}$ Vgl. Shleifer (2005).

${ }^{3}$ Vgl. Stigler (1975), Posner (1975) und Friedman (2002).

${ }^{4} \mathrm{Vgl}$. Akerlof et al. (2009).

${ }^{5}$ Neal et al. (2005) zeigen auf, dass Finanzmärkte mit wenig staatlicher Intervention und Überwachung entstanden. Glaeser et al. (2001) vergleichen die Anzahl der Initial Public Offerings (IPOs) der streng regulierten Börse in Warschau (136 IPOs) gegenüber der wenig regulierten Börse in Prag (keine IPOs) in den Jahren von 1991-1998. Die Autoren folgern daraus, dass ein hohes Maß an Regulierung zum Zweck des Investorenschutzes die Entwicklung eines Handelsplatzes beflügelt. Stringham et al. (2008) untersuchen ebenfalls diese beiden Börsen, kommen jedoch zu einem entgegengesetzten Ergebnis. Demnach hat der tschechische Staat die Börse in Prag an seiner Entfaltung gehindert.

${ }^{6}$ Nach Shleifer (2005) ist ein Versäumnis der Einhaltung staatlich festgelegter Regeln für ein Gericht viel leichter zu durchschauen als eine Nichterfüllung individueller Vereinbarungen.

${ }^{7}$ Vgl. Europäische Kommission (2002a). Vgl. De Haan et al. (2009).

${ }^{8}$ Eine Regulierung ist für alle Mitgliedstaaten direkt bindend und bedarf keiner Transposition in die jeweilige nationale Gesetzgebung. Eine Regulierung erzwingt somit eine vollständige Integration. Die Bestimmungen sind einheitlich und überstimmen die nationale Gesetzgebung. Vgl. De Haan et al. (2009), S. 40-41.

${ }^{9}$ Eine Direktive ist für die Mitgliedsstaaten ebenfalls bindend, gibt den nationalen Autoritäten jedoch die Wahl der Form und Methode. Direktiven müssen in einem Zeitrahmen von gewöhnlich 18 bis 24 Monaten in nationales Recht eingegliedert werden. Eine Direktive lässt Spielraum für Unterschiede bei der Implementierung. Vgl. De Haan et al. (2009).
} 
genden durch die englische Abkürzung MiFID - Markets in Financial Instruments Directive


päischen Wertpapiermärkte seit nun sechs Jahren.

In der öffentlichen Diskussion reichen die Bewertungen der MiFID vom Eigenlob der Europäischen Kommission („Ground breaking piece of legislation“"11) bis hin zu polemischen Äußerungen in der Finanzpresse wie "Miffed with MiFID“12 oder "A nightmarish, alien creature capable of inflicting a terrible financial sting on all it touches" ${ }^{\prime 13}$. Aktuell (Dezember 2013) wird die Überarbeitung der MiFID als MiFID II bzw. MiFID Review diskutiert und soll nach Abschluss des europäischen Gesetzgebungsprozesses frühestens ab 2016 anwendbar sein. Trotz dieser weitreichenden Überarbeitung unter anderem im Hinblick auf Transparenzvorschriften und Marktstruktur fehlt eine systematische Analyse der MiFID bezüglich der Zielerreichung in den vergangenen sechs Jahren.

Ziel dieses Artikels ist es daher, erstmalig die Erreichung der MiFID-Ziele anhand einer systematischen Analyse der akademischen Literatur zu bewerten. Hierzu werden zunächst in Abschnitt 2 die MiFID-Ziele identifiziert und zueinander in Beziehung gesetzt. In Abschnitt 3 wird dargelegt, welche Instrumente zur Zielerreichung eingeführt bzw. angedacht wurden. Darauf aufbauend werden in Abschnitt 4 unter Anwendung der wissenschaftlichen Methode des systematischen Reviews die relevanten akademischen Artikel identifiziert, ihre methodischen und inhaltlichen Aspekte in einer Metaanalyse aggregiert sowie die Aussagen der einzelnen Beiträge betreffend der Zielerreichung analysiert. Schließlich werden die Erkenntnisse in Abschnitt 5 zusammengefasst und in die aktuelle Diskussion eingebunden.

\section{Die Ziele der MiFID}

\subsection{Identifizierung der MiFID-Ziele}

Am 19. November 2002 veröffentlichte die Europäische Kommission ihren Vorschlag für eine neue Direktive über Wertpapierdienstleistungen und geregelte Märkte. Darin wird als allge-

\footnotetext{
${ }^{10}$ Vgl. Europäisches Parlament (2004).

${ }^{11}$ Europäische Kommission (2006b).

${ }^{12}$ Sell (2006).

${ }^{13}$ Dickson (2005).
} 
meine Zielsetzung die Gewährleistung der Integrität und die Förderung transparenter, effizienter und integrierter Finanzmärkte mittels der Entwicklung grundlegender Regeln fixiert. ${ }^{14}$ In den weiteren Ausführungen betont die Europäische Kommission die Notwendigkeit „die europäischen Wertpapiermärkte wettbewerbsfähiger [zu] machen ${ }^{\prime 15}$ mit der Absicht „Wertpapiere mit einem Maximum an Effizienz und einem Minimum an Kosten zu handeln ${ }^{16}$. Geringe Handelskosten und Effizienz sollen erzielt werden, indem Handelsplattformen mit den niedrigsten Handelskosten mit einem größeren Handelsvolumen belohnt werden. ${ }^{17}$

Das Regulierungsziel der MiFID ist somit Effizienz in Form reduzierter Kosten, wobei Effizienz eine idealtypische konzeptionelle Situation darstellt und Kosten als quantifizierbare Größen gelten. Wettbewerb, Integration, Transparenz und Integrität fungieren als Zwischenziele, die auf das angestrebte Ziel der Effizienz bzw. Kostenreduzierung ${ }^{18}$ einwirken. Ein Zwischenziel kann als eine Variable verstanden werden, die einerseits in einem engen Zusammenhang zum wirtschaftspolitischen Instrumenteneinsatz und andererseits zum anvisierten Ziel steht. ${ }^{19}$ Die MiFID soll Finanzintermediäre und Handelsplätze zu Effizienz drängen, damit die Handelskosten für Investoren sinken.

\subsection{Die MiFID-Ziele: Effizienz und Kostensenkung}

Die Kriterien zur Beurteilung der Effizienz von Wertpapiermärkten gliedern sich in der wissenschaftlichen Literatur in die operative Effizienz, die Informationseffizienz, die Bewertungseffizienz und die allokative Effizienz. ${ }^{20}$ Die operative Effizienz ist die Annäherung an das Ideal des friktionslosen Handels. Hierzu sollen Kosten für Infrastruktur sowie Inanspruchnahme von Finanzintermediären minimiert und der Handel weitgehend automatisiert werden.

\footnotetext{
${ }^{14}$ Vgl. Europäische Kommission (2002a), S. 9.

${ }^{15}$ Europäische Kommission (2002b).

${ }^{16}$ Europäische Kommission (2002b).

${ }^{17} \mathrm{Vgl}$. Europäische Kommission (2002b).

${ }^{18}$ Die Europäische Kommission hat im Vorfeld der Veröffentlichung des MiFID-Vorschlages eine Studie in Auftrag gegeben, um das Potenzial zur Senkung der Kapital- und Handelskosten auszuloten. Der Studie von London Economics (2002) zufolge können durch die Integration europäischer Finanzmärkte die expliziten Handelskosten um 11\%, die impliziten Handelskosten um $8 \%$ und die Eigenkapitalkosten um 0,5 \% gesenkt werden.

${ }^{19} \mathrm{Vgl}$. Ramb (1987).

${ }^{20}$ Vgl. Bienert (1996).
} 
Die Informationseffizienz ist die Angleichung der Kurse an den Zustand vollkommener Information. Ein Marktplatz gilt nach Fama (1970) als informationseffizient, wenn Preise immer vollständig alle verfügbaren Informationen wiederspiegeln. ${ }^{21}$ Jensen (1978) bewertet einen Markt als informationseffizient, wenn sich anhand von Informationen keine Handelsgewinne mehr realisieren lassen. Damit es jedoch überhaupt einen Anreiz zum Handeln gibt, muss es in der Realität einen Grad an Informationsineffizienz geben. Diesen Zusammenhang bezeichnen Grossman et al. (1980) als Informationsparadoxon. Daher ist ein Maß an Transparenz erforderlich, das einerseits das Problem asymmetrisch verteilter Informationen minimiert und andererseits den Anreiz für Marktteilnehmer aufrechterhält, von der Informationsbeschaffung zu profitieren.

Die Bewertungseffizienz ist das Ergebnis eines Vergleiches zweier Zustände. An einem bewertungseffizienten Markt stimmen Kurse und Fundamentalwerte überein. ${ }^{22}$ Der Fundamentalwert entspricht dabei dem Barwert aller zukünftig erwarteten Zahlungsströme. ${ }^{23}$ Marktpreise offenbaren das Wissen und die Wünsche einer Vielzahl von Individuen und ermöglichen so die Koordination bester Nutzungsmöglichkeiten wirtschaftlicher Ressourcen ${ }^{24}$, die im Fokus der Allokationseffizienz steht.

Unter Allokationseffizienz versteht man die Koordination der Ressourcen auf konkurrierende Verwendungsmöglichkeiten, wobei der maximale gesellschaftliche Nutzen ausgeschöpft werden soll. ${ }^{25}$ Die Beurteilung der Allokationseffizienz wirft die Frage auf, ob die erzielten Ergebnisse die Besten waren, die sich auf der Informationsbasis erreichen ließen, die zum Zeitpunkt der Entscheidung verfügbar war oder hätte verfügbar gemacht werden können. ${ }^{26}$ Es werden keine Resultate sondern der Prozess der Ressourcenverteilung analysiert. Die allokative Effizienz ist demnach nicht die Eigenschaft eines Ergebnisses, sondern einer Regel.

\footnotetext{
${ }^{21}$ Die Beschreibung der Informationseffizienz eines Marktes erfolgt nach Fama (1970) in Abhängigkeit der Informationsverbreitung nach den drei Kategorien schwach, mittelstreng und streng. In der schwachen Form reflektiert der aktuelle Marktpreis historische Kursdaten. In der mittelstrengen Variante sind alle öffentlich zugänglichen Informationen im Preis enthalten. Die strenge Informationseffizienz berücksichtigt zudem Insiderinformationen. Die Kategoriesierung der Informationseffizienz nach Fama ist ein theoretisches Konzept. Als Unterscheidungskriterium für empirische Untersuchungen bieten sich daher die Grenzkosten der Informationsbeschaffung an. Neumann et al. (1982) unterscheiden nach der Verfügbarkeit vorhandener Informationen in zentral veröffentlichte Informationen (Grenzkosten nahe Null), dezentral veröffentlichte Informationen und vorübergehend monopolisierte Informationen.

${ }^{22} \mathrm{Vgl}$. Bienert (1996).

${ }^{23} \mathrm{Vgl}$. Tobin (1984).

${ }^{24} \mathrm{Vgl}$. Hayek (1945).

${ }^{25}$ Vgl. Beckmann (1998).

${ }^{26} \mathrm{Vgl}$. Friend (1972).
} 
Allokationsregeln dienen als Kriterium für die Bestimmung der allokativen Effizienz eines Marktes. ${ }^{27}$

\subsection{Die MiFID-Zwischenziele: Wettbewerb, Integration, Transparenz}

\section{und Integrität}

\subsubsection{Wettbewerb}

Auf der Basis der Argumentation, dass Handelsplätze natürliche Monopol seien und Liquidität ${ }^{28}$ eine positive Externalität darstelle, hatten einige Mitgliedsstaaten der EU den Wettbewerb im Wertpapierhandel eingeschränkt. Baumol (1982) beschreibt in seinem Modell der bestreitbaren Märkte (Contestable Markets ${ }^{29}$ ), dass monopolähnliche Marktstrukturen nicht zwangsläufig reguliert werden müssen. Hit-and-Run-Raider sorgen für Wettbewerb, indem sie im Fall von Preiserhöhungen sofort als potenzielle Rivalen in den Markt treten, um Überrenditen zu vereinnahmen. Die bloße Gefahr eines Markteintritts hält deswegen die existierenden Anbieter davon ab, Monopolpreise zu verlangen. Darüber hinaus zeigen empirische Beispiele, dass Regulierung, die unter dem Vorwand der Protektion der Öffentlichkeit eingeführt wurde, sich zum Schutzorgan für Monopole und Kartelle gegenüber Konsumenten und Konkurrenzunternehmen wandelt. ${ }^{30}$ Die Regulierung natürlicher Monopole kreiert Markteintritts- und Austrittsbarrieren und schützt Monopolerträge.

Auf der Seite der Nachfrage spielen Netzwerkeffekte eine wichtige Rolle. Ein Wertpapiermarkt ist umso liquider, je mehr Teilnehmer diesen nutzen. ${ }^{31}$ Aufgrund dieser positiven Externalität weist der Wertpapierhandel die natürliche Tendenz auf, sich am Ort des größten Umsatzes zu konzentrieren. ${ }^{32}$ Das Argument den Handel auf einem Marktplatz zentrieren zu

\footnotetext{
${ }^{27}$ Vgl. Holmström et al. (1983).

${ }^{28}$ Liquidität im Wertpapierhandel ist die Fähigkeit einerseits ohne zeitliche Verzögerung und andererseits ohne Preisrisiko ein Asset in Bargeld zu wandeln. Vgl. Tinic et al. (1979).

${ }^{29}$ Ein Markt ist perfekt bestreitbar, wenn Neulinge Märkte frei (freier Zugriff auf die gleiche, ggf. kostenintensive Technologie) betreten und kostenlos (Abwesenheit von Sinkkosten) wieder verlassen können.

${ }^{30}$ Stigler (1975) führt die durch die SEC gesetzlich geschützten ehemals hohen Brokergebühren an der New York Stock Exchange (NYSE) als Beispiel an. An die Stelle eines aufgrund der Mindestpreise nicht vorhandenen Preiswettbewerbs trat der Service-Wettbewerb (auch unter der Bezeichnung "Soft-Dollars" bekannt). Die Fixed Comissions wurden im Jahr 1975, dem Jahr der Buchveröffentlichung Stiglers, im Rahmen der Verabschiedung der Securities Act Amendments aufgehoben. Vgl. Stoll (2003). Die Dienstleistungen der SoftDollars können nicht als gleichwertige Kompensation angesehen werden, welche den Verlust der Konsumentenrente durch fehlenden Preiswettbewerb ausgleichen. Vgl. Steil et al. (2003).

${ }^{31}$ Vgl. Petrella (2010).

${ }^{32}$ Vgl. Pagano (1989). Vgl. Chowdhry et al. (1991).
} 
müssen, hat aufgrund der technischen Möglichkeiten der Integration der Liquidität (zum Beispiel über Smart Order Routing (SOR) oder die Integration fragmentierter Orderbücher auf dem Handels-Front-End der Marktteilnehmer) an Bedeutung verloren.

\subsubsection{Integration}

Der Wettbewerbsdruck auf Handelsplätze und Intermediäre wird durch die Förderung der Integration europäischer Finanzmärkte weiter erhöht. ${ }^{33}$ Die MiFID bezieht die Forderung nach Integration auf die technische Infrastruktur im Wertpapierhandel. ${ }^{34}$ Lee (1993) versteht unter der technischen Integration im Wertpapierhandel den vollständigen, zeitnahen und marktübergreifenden Austausch anhand miteinander kommunizierender elektronischer Verbindungen. Die technische Integration soll der Fragmentierung der Liquidität, die durch Wettbewerb hervorgerufen wurde, entgegenwirken. Fragmentierung tritt auf, wenn Aufträge für gleiche Wertpapiere an unterschiedliche Marktplätze gerichtet werden und nicht miteinander interagieren. ${ }^{35}$ In einem voll integrierten Markt haben hingegen Aufträge über alle physischen Handelsplätze hinweg die Möglichkeit gegen die besten Quotierungen ausgeführt zu werden.

\subsubsection{Transparenz}

Die Realisierung der positiven Wirkung von Wettbewerb und Integration auf die Handelskosten erfordert die Offenlegung von Informationen. Ohne die Kenntnis der Lokation des besten Preises wird ein Anleger den Markt der Erstnotiz bevorzugen. ${ }^{36}$ Ein weiteres Zwischenziel der EU ist es daher, die potentiell negativen Effekte der fragmentierten Liquidität durch erhöhte Transparenz auszugleichen.

Der Wertpapierhandel unterscheidet Transparenz bezüglich der Komponenten Vorhandelsund Nachhandelstransparenz. Vorhandelstransparenz ist die Fähigkeit, die Handelsinteressen (Wertpapierorders) anderer Marktteilnehmer zu beobachten. ${ }^{37}$ Nachhandelstransparenz bezieht sich auf die Offenlegung von Informationen über abgeschlossene Geschäfte. ${ }^{38}$ Ziel

\footnotetext{
${ }^{33}$ Vgl. Giannetti et al. (2002).

${ }^{34} \mathrm{Vgl}$. Europäische Kommission (2002a).

${ }^{35}$ Vgl. Stoll (2003).

${ }^{36}$ Aus diesem Grund schirmte die NYSE ursprünglich die Preise vor der Öffentlichkeit ab. Vgl. Stoll (2003).

${ }^{37}$ Vgl. Boehmer et al. (2005).

${ }^{38}$ Vgl. Porter et al. (1998).
} 
der Transparenz ist es darüber hinaus, Suchkosten für die Identifikation eines handelswilligen Partners zu reduzieren, Aktienmärkte miteinander vergleichbar zu machen, die Orderausführung zu kontrollieren und die Preisfindung zu beschleunigen.

\subsubsection{Integrität}

Wettbewerb, Integration und Transparenz senken Handelskosten. Ob jedoch Investoren von der Kostenreduzierung profitieren oder diese von den beauftragten Intermediären abgeschöpft wird, steht im Zentrum der Integrität. Aufgabe von Finanzintermediären ist es, die Vermittlung zwischen Käufer und Verkäufer zu erleichtern, sowie die Investoren bei diesem Prozess zu beraten. ${ }^{39}$ Den Begriff der Integrität umschreibt Levitt (1999) als „a promise that brokers will act in their customers' best interest". Im Kern verkauft der Broker seinem Kunden direkt oder indirekt Informationen. ${ }^{40}$ Der strukturelle Konflikt in der Investor-BrokerBeziehung entsteht aufgrund der asymmetrischen Verteilung von Informationen zugunsten des Intermediäres. Hierdurch ist der Anleger mit einer bestimmten Wahrscheinlichkeit einem opportunistischen Verhalten der Intermediäre ausgesetzt.

\subsection{Die Wirkung der MiFID-Zwischenziele auf die MifID-Ziele}

Die vier MiFID-Zwischenziele Wettbewerb, Integration, Transparenz und Integrität wirken auf die unterschiedlichen Effizienzformen. Das Zwischenziel Wettbewerb dient in erster Linie einer effizienten operativen Durchführung des Wertpapierhandels. Integration wirkt der durch Wettbewerb verursachten Fragmentierung der Liquidität entgegen und verbessert dadurch die operative Effizienz. In Verbindung mit Transparenz hilft Integration die fragmentierten Informationen wieder zusammenzuführen. Transparenz reduziert die durch asymmetrische Informationsverteilung verursachten Risiken und sichert dadurch die Informationseffizienz. Die Allokationsregel strebt nach einem allokativ effizienten Zustand, um einen wohlfahrtsmaximierenden Handelsaustausch zu erreichen, der versucht, einige Marktteilnehmer besser zu stellen ohne andere Marktteilnehmer dabei zu benachteiligen. ${ }^{41}$ Diesen Konflikt zwischen Käufern, Verkäufern und Intermediären im Wertpapierhandel auszugleichen, ist Aufgabe der Integrität.

\footnotetext{
${ }^{39}$ Zu weiteren Funktionen von Intermediären siehe Levine (1997).

${ }^{40} \mathrm{Vgl}$. Pacces (2000).

${ }^{41} \mathrm{Vgl}$. Holmström et al. (1983).
} 
Das messbare Kriterium zur Effizienzermittlung sind Kosten, welche den Grad der Annäherung an die vollkommenen Effizienzformen aufzeigen. Eine Reduzierung der expliziten Handelskosten wird einem erhöhten Wettbewerb zugeschrieben. ${ }^{42}$ Ferner senkt Integration die impliziten Handelskosten, weil eine größere Anzahl an Marktteilnehmern die Liquidität erweitert und mehr Informationen aggregiert werden können. Uninformierte Anleger beziehen in ihre Handelsabsichten einen Risikopuffer ein, der sich in einer erweiterten Geld-BriefSpanne ausdrückt, um sich gegen den Verlust aus dem Handel mit einer informierten Gegenpartei zu schützen. ${ }^{43}$ Das richtige Maß an Transparenz senkt diese Informationsrisikokosten.

Die expliziten und impliziten Handelskosten inklusive der Informationsrisikokosten fließen in die Kapitalkosten ein, die der Wertpapierbewertung zugrundeliegen. ${ }^{44}$ Investoren wollen im Gleichgewicht für Handelskosten kompensiert werden. Amihud et al. (2006) belegen empirisch, dass Handelskosten Anleger veranlassen, höhere Renditeforderungen zu stellen. Die Autoren nehmen an, dass die Reduzierung von Handelskosten die Kapitalkosten senkt.

Schließlich soll das Zwischenziel der Integrität Handelskosten reduzieren. Maßnahmen der Integrität dienen dem Schutz der Anleger und beseitigen die in einem Modell von Easley et al. (2009) definierten Kosten der Ambiguität. Die Kosten der Ambiguität verringern die Liquidität im Sinne der Unterlassung ökonomisch sinnvoller Handelsaktivitäten aufgrund von Unsicherheit unter den Investoren. Abbildung 1 stellt den Zusammenhang der MiFID Ziele und der verschiedenen Kostenaspekte dar.

\footnotetext{
${ }^{42}$ Für Angaben bezüglich der Senkung expliziter Handelskosten siehe beispielsweise Gentile et al. (2011).

${ }^{43}$ Vgl. Akerlof (1970). Vgl. Glosten et al. (1985). Vgl. Leuz et al. (2008).

${ }^{44} \mathrm{Vgl}$. Amihud et al. (1986). Lambert et al. (2012) untersuchen die Beziehung asymmetrisch verteilter Informationen und Kapitalkosten. Butler et al. (2005) belegen empirisch, dass Liquidität die Kosten der Kapitalbeschaffung senkt.
} 


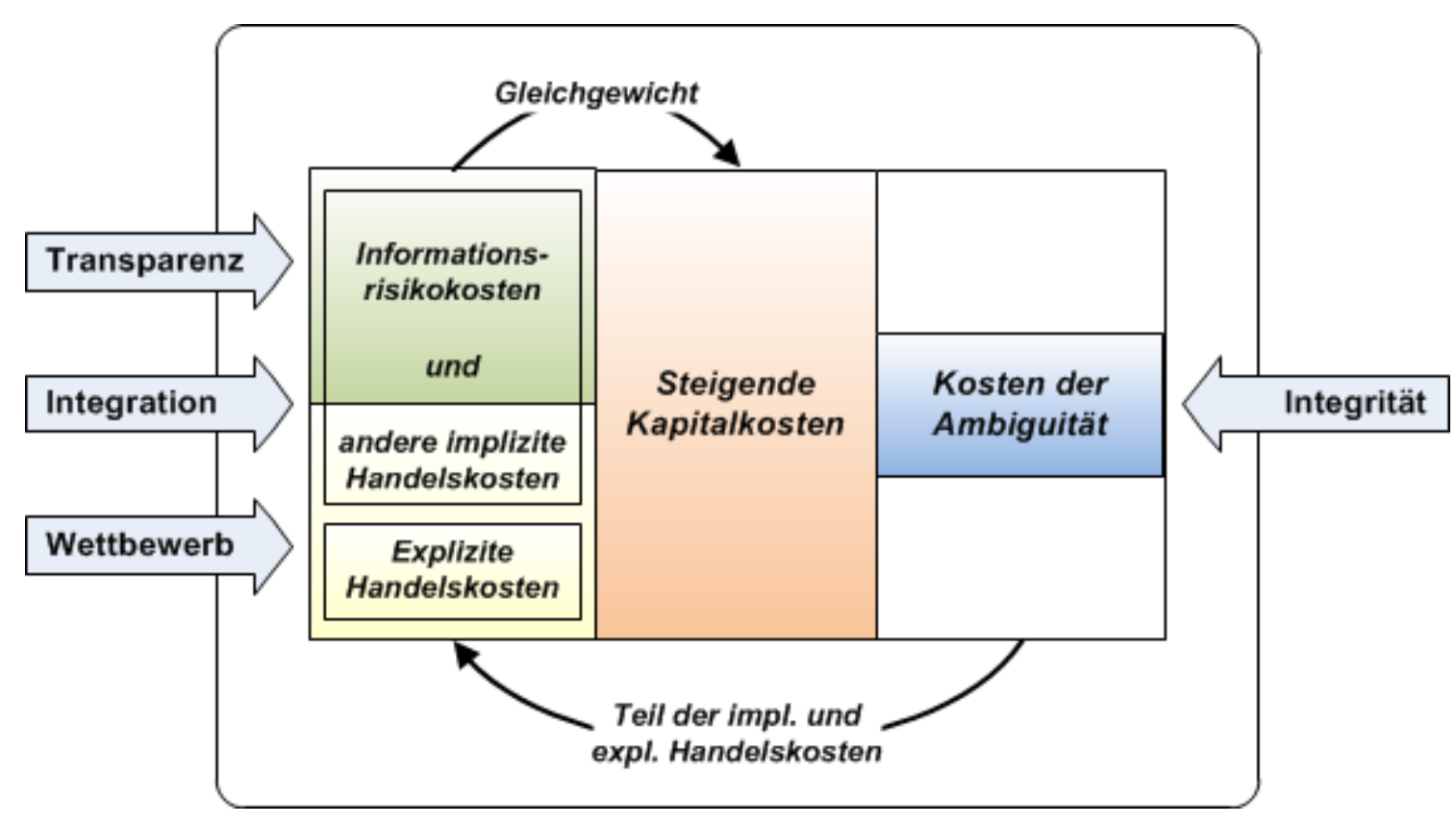

Abbildung 1: MiFID-Ziele - Kostenaspekte (Eigene Darstellung)

Die Erfassung der verschiedenen Handelskosten ist in der Realität unterschiedlich komplex. Explizite Handelskosten sind leicht zu ermitteln, da sie von Intermediären und Marktplätzen offengelegt werden. Implizite Kosten können zwar gemessen werden, eine kausale Zuordnung ist hingegen schwierig. So könnte eine Verbesserung der Liquidität auf den Wettbewerb unter Liquiditätsspendern, auf die Integration von Marktplätzen, auf eine Reduzierung der Informationsrisikokosten oder eine Steigerung des Handelsvolumens aufgrund der Stärkung der rechtlichen Position der Investoren zurückgeführt werden.

\section{Die Instrumente zur MiFID-Zielerreichung}

\subsection{Wettbewerb über die Aufhebung der Konzentrationsregel}

Die Europäische Kommission hat freien Wettbewerb im Wertpapierhandel durch die Abschaffung nationaler Börsenvorränge bzw. Börsenzwänge ${ }^{45}$ und durch die Zulassung alternativer Handelsplätze in allen Mitgliedstaaten forciert. In erster Linie soll der Wettbewerb

\footnotetext{
${ }^{45}$ Vgl. Europäische Kommission (2002a) und Europäisches Parlament (2004) Artikel 69.
} 
„Zwischen traditionellen Börsen und anderen Handelssystemen ${ }^{446}$ gefördert werden. Die alternativen Formen der Orderausführung werden häufig von Finanzintermediären betrieben. Die traditionelle Trennung der Verantwortungsbereiche der Marktplätze einerseits und der Intermediäre andererseits werden durch die MiFID somit aufgeweicht.

\subsection{Integration der fragmentierten Liquidität und}

\section{Handelsinformationen}

Die technische Integration von Marktplätzen kann der Fragmentierung von Liquidität und Informationen entgegenwirken. Die Fragmentierung der Liquidität kann durch ein TradeThrough-Verbot verhindert werden. Unter einem Trade-Through versteht man die Ausführung eines Geschäfts am gegenwärtigen Markt, obwohl zu diesem Zeitpunkt an einem anderen Markt ein besserer Preis erzielbar wäre. ${ }^{47}$ In den USA ist das Trade-Through-Verbot an die Verpflichtung gekoppelt, Quotierungen unverzüglich und automatisiert zugänglich zu machen.$^{48}$ Dies schützt den Anleger nicht nur vor einem ungünstigen Preis, sondern reduziert auch die Gefahr verpasster Handelschancen für Limit-Aufträge. Die MiFID sieht jedoch keine Notwendigkeit Trade-Throughs zu verbieten und Marktplätze zur Integration der Liquidität zu verpflichten.

Die zweite Komponente der Integration betrifft die Aggregierung von Handelsinformationen anhand eines zentralen Informationssystems, das einerseits die Quotierungen und andererseits die Geschäfte aller Handelssysteme in einem einheitlichen Format empfängt, zentral konsolidiert und den Marktteilnehmern zur Verfügung stellt. Ein aus den Quotierungen entstehendes Consolidated Quotation System (CQS) veröffentlicht in den USA die Spitze des Orderbuchs, also das Best Bid und Best Offer (BBO). Demgegenüber stellt ein Consolidated Tape System (CTS) ein Berichtswesen dar, welches Marktdaten über abgeschlossene Geschäfte verschiedener Handelsplätze weiterverteilt und somit keine Indikation liefert, an welchen Orten es beste Handelsmöglichkeiten gibt. Ein CTS und ein CQS übermitteln Transaktionen und Quotierungen an eine zentrale Stelle, führen selbst aber keine Aufträge aus.

\footnotetext{
${ }^{46}$ Vgl. Europäische Kommission (2002b).

${ }^{47}$ In den USA soll die Order Protection Rule (Rule 611) Trade-Throughs verhindern. Vgl. U.S. Securities and Exchange Commission (2005).

${ }^{48}$ Rule 610. Vgl. U.S. Securities and Exchange Commission (2005).
} 
Eine vollständige Integration der Märkte setzt die Aggregierung der Informationen voraus. Die MiFID beschreibt ausführlich die Notwendigkeit Kenntnisse über „Einzelheiten zu aktuellen Möglichkeiten des Handels" 49 zu erlangen, um "eine effektive Integration der Aktienmärkte der Mitgliedstaaten sicherzustellen, die Effizienz des globalen Kursbildungsprozesses bei Eigenkapitalinstrumenten zu steigern und die effektive Einhaltung der Pflicht zur „bestmöglichen Ausführung" zu fördern" ${ }^{50}$, ohne jedoch den Begriff des CQS explizit zu nennen. Die Möglichkeit der Integration der Handelsinformationen anhand eines CTS und CQS, die man aus den US-Märkten kennt, wird von der MiFID nicht umgesetzt, ein europäisches CTS wird jedoch aktuell im Kontext der MiFID II diskutiert.

\subsection{Transparenz über die Offenlegung der Liquidität der}

\section{Handelsplätze}

Die MiFID klassifiziert die verschiedenen Mechanismen der Orderausführung in geregelte Märkte und MTFs, die multilaterale Handelsplätze darstellen, sowie in Systematische Internalisierer $(\mathrm{SI})^{51}$ und Over-The-Counter-Geschäfte (OTC) ${ }^{52}$, die als bilaterale Ausführungen gelten. Multilaterale Handelsplätze müssen die aggregierten Quotierungen der jeweils fünf besten Kauf- und Verkaufsaufträge sowie das entsprechende Volumen veröffentlichen. ${ }^{53}$ Ausnahmen für die Vorhandelstransparenz sollen nur für bestimmte Handelsgeschäfte gelten. ${ }^{54}$ Die Vorschriften zur Vorhandelstransparenz stellen für geregelte Märkte keine Neuerung dar, da die Veröffentlichung von Quotierungen bereits praktiziert wird. ${ }^{55}$

\footnotetext{
${ }^{49}$ Europäisches Parlament (2004) Erwägungsgrund 44.

${ }^{50}$ Europäisches Parlament (2004) Erwägungsgrund 44.

${ }^{51}$ Die MiFID definiert einen Systematischen Internalisierer als „eine Wertpapierfirma, die in organisierter und systematischer Weise häufig regelmäßig Handel für eigene Rechnung durch Ausführung von Kundenaufträgen außerhalb eines geregelten Marktes oder eines MTF treibt". Vgl. Europäisches Parlament (2004) Artikel 4, Absatz 1, Punkt 7.

52 OTC Geschäfte werden als vierte Form des Aktienhandels in Erwägungsgrund 53 genannt. Vgl. Europäisches Parlament (2004).

53 Bezüglich MTFs vgl. Europäisches Parlament (2004) Artikel 29 (1) und bezüglich Geregelter Märkte Artikel 44 (1).

${ }^{54}$ Vgl. Europäisches Parlament (2004). Hierzu gehören Aufträge die einen marktüblichen Geschäftsumfang (Artikel 22, Absatz 2) übersteigen (Geregelte Märkte: Artikel 44 \& 45, Absatz 2; MTFs: Artikel 29 \& 30, Absatz 2) oder zu einem Referenzpreis (Geregelte Märkte: Artikel 44, Absatz 3, Buchstabe c; MTFs: Artikel 29, Absatz 3, Buchstabe c) ausgeführt werden. Der marktübliche Geschäftsumfang wird von der ESMA je Aktie jährlich bestimmt und veröffentlicht.

${ }^{55}$ Abgeschlossene Geschäfte sind unter Angabe des Marktplatzes, des Preises, des Volumens und des Zeitpunkts des Abschlusses zu veröffentlichen. Diese Informationen sind den Marktteilnehmern innerhalb einer Frist von maximal drei Minuten anzuzeigen.
} 
SIs werden verpflichtet, für liquide Aktien verbindliche Preisofferten in standardmäßigen Marktgrößen während der üblichen Geschäftszeiten regelmäßig und kontinuierlich zu veröffentlichen. ${ }^{56}$ OTC Geschäfte sind hingegen an keine Vorhandelstransparenz gebunden und die Veröffentlichung abgeschlossener Geschäfte kann abhängig vom Transaktionsvolumen von 60 Minuten bis zum Ende des 3. Handelstages nach der Transaktion hinausgezögert werden. ${ }^{57}$

\subsection{Integrität über das Best Execution Konzept}

Best Execution ist der Ansatz, dass Intermediäre im Interesse des Kunden bei der Auftragsausführung agieren. Die MiFID beabsichtigt, die sehr heterogenen nationalen Regularien zur Best Execution zu harmonisieren und wählt gegenüber der präskriptiven und auf den Ausführungspreis fokussierten US-amerikanischen Variante eine eher konzeptionelle Herangehensweise, die mehrere Aspekte über den Preis hinaus (u.a. Kosten, Schnelligkeit und Wahrscheinlichkeit der Ausführung) hervorhebt. ${ }^{58}$ Die beste Orderausführung wird in der die MiFID ergänzenden Durchführungsrichtlinie auf der zweiten Ebene des europäischen Rechtsetzungsverfahrens für Privatanleger dahingehend konkretisiert, dass der Ausführungspreis inklusive der expliziten Handelskosten (Gesamtentgelt) die erste Priorität bei der Bewertung einer Orderausführung erhält. ${ }^{59}$ Inwieweit ein dynamisches, die aktuelle Marktsituation und individuelle Order betrachtendes oder ein statisches, rein auf historischen generischen Daten basierendes Modell der Orderausführung in der Best Execution Anwendung findet, bleibt dem Broker überlassen.

\section{Die Bewertung der MiFID-Zielerreichung}

\subsection{Systematischer Review und Metaanalyse}

In der akademischen Forschung entwickelt sich die Literaturanalyse zunehmend zu einer eigenständigen wissenschaftlichen Methode. ${ }^{60}$ Der systematische Review und die anschlie-

\footnotetext{
${ }^{56}$ Vgl. Europäisches Parlament (2004) Artikel 27, Absatz 1.

${ }^{57}$ Vgl. Rößner (2008).

${ }^{58}$ Vgl. Europäisches Parlament (2004) Artikel 21, Absatz 1.

${ }^{59}$ Vgl. Europäische Kommission (2006a), Erwägungsgrund 67.

${ }^{60} \mathrm{Vgl}$. Fettke (2006).
} 
ßende Metaanalyse haben das Ziel, einen ausgewogenen und umfassenden Überblick über eine spezifische Problemstellung zu erlangen bzw. aus dem identifizierten Datenbestand inhaltliche Aggregate zu bilden. Fink definiert den systematischen Review als „a sytematic, explicit, and reproducible method for identifying, evaluating, and interpreting the existing body of work produced by researches and scholars" ${ }^{61}$. Dabei gilt es, sich auf einen eingegrenzten Themenbereich und eine konkret formulierte Fragestellung zu konzentrieren. Die systematische und explizite Methode unterliegt einer definierten Schrittfolge, die sich von der Fragestellung über die Literaturrecherche und Literaturauswertung bis zur Präsentation der Ergebnisse erstreckt. ${ }^{62}$

Als ein anwendbares Konzept zur Qualitätswahrung eines systematischen Reviews können die in der medizinischen Forschung entwickelten Leitlinien der Preferred Reporting Items for Systematic Reviews and Meta-Analyses (PRISMA) herangezogen werden. PRISMA ist ein nachweisorientiertes Instrument, welches in der medizinischen Behandlung helfen soll, Entscheidungen auf der Basis wissenschaftlich empirisch nachgewiesener Wirksamkeit zu treffen. ${ }^{63}$ In der wissenschaftlichen Literaturrecherche medizinischer Fachjournale fest verankert, lässt sich das Verfahren auch auf andere akademische Disziplinen wie die Wirtschaftswissenschaften übertragen.

Insgesamt wurden in der vorliegenden Untersuchung 27 Artikel für den systematischen Review und die Meta-Analyse berücksichtigt (s. Abbildung 2). ${ }^{64} \mathrm{Im}$ Rahmen der Identifikation wissenschaftlicher Artikel erfolgte zunächst eine Schlüsselwort-basierte Suche mit dem Ausdruck „MiFID“ in den Online-Literaturdatenbanken EBSCO, Science Direct, Emerald, Springer, JSTOR, Journals Cambridge, Wiley und Social Science Research Network (SSRN) im Zeitraum vom 1. Januar 2007 bis zum 31. Januar 2013. Die Recherche erstreckte sich jeweils über das ganze Dokument mit der Ausnahme der Datenbanken von Springer und SSRN, bei denen die Stichwort-Suche auf Titel und Abstract begrenzt war. Ferner beschränkte sich die Suche auf wissenschaftliche Artikel.

Die identifizierten 447 Artikel wurden einem groben Screening unterzogen. Eliminiert wurden nicht englisch- oder deutschsprachige Publikationen, mehrfach erfasste Artikel oder Ar-

\footnotetext{
${ }^{61}$ Fink (1998), S. 37.

${ }^{62} \mathrm{Vgl}$. Fettke (2006).

${ }^{63}$ Vgl. Moher et al. (2009).

${ }^{64}$ Die meisten Beiträge für den systematischen Review lieferte mit 15 Artikeln die Literaturdatenbank SSRN.
} 
tikel mit einer anderen Verwendung des Akronyms "MiFID“65. In einem detaillierten Screening nach inhaltlichen Kriterien wurden die Abstracts und die relevanten Textpassagen dahingehend evaluiert, ob sie sich sowohl mit den MiFID-Zielen auseinandersetzen als auch diese explizit oder implizit bewerten. Die Analyse empirischer Studien selektierte nur die Artikel, deren Datenbasis (auch) aus Daten der Zeit seit der Einführung der MiFID am 01. November 2007 (Post-MiFID Periode) besteht. Die Qualifikationsanforderungen wurden von 21 Artikeln erfüllt.

Als Ausnahmen wurden sechs weitere Artikel mit in die zu untersuchende Literaturmenge aufgenommen. ${ }^{66}$ Untersuchungen zu Inducements (Assmann 2007) und zu Trade-Throughs (Ende et al. (2010)), eine empirische Auswertung der Best Execution (Gomber et al. 2012b) sowie weitere Artikel auf dem Gebiet der Fragmentierung des Wertpapierhandels (Degryse et al. (2011), Gomber et al. (2011a)), erweitern das Themenspektrum. Schließlich konnten anhand der Überprüfung der Literaturlisten der einzelnen Beiträge keine im Rahmen des Prozesses ausgelassenen Artikel gefunden werden.

\footnotetext{
${ }^{65}$ Beispiel: Membrane Introduction Flame Ionization Detection (MIFID).

${ }^{66}$ Der Artikel von Chlistalla et al. (2011) wäre im systematischen Review erfasst worden, wenn sich die Schlüsselwort-basierte Suche in der Springer-Datenbank nicht auf den Titel und Abstract beschränkt hätte.
} 


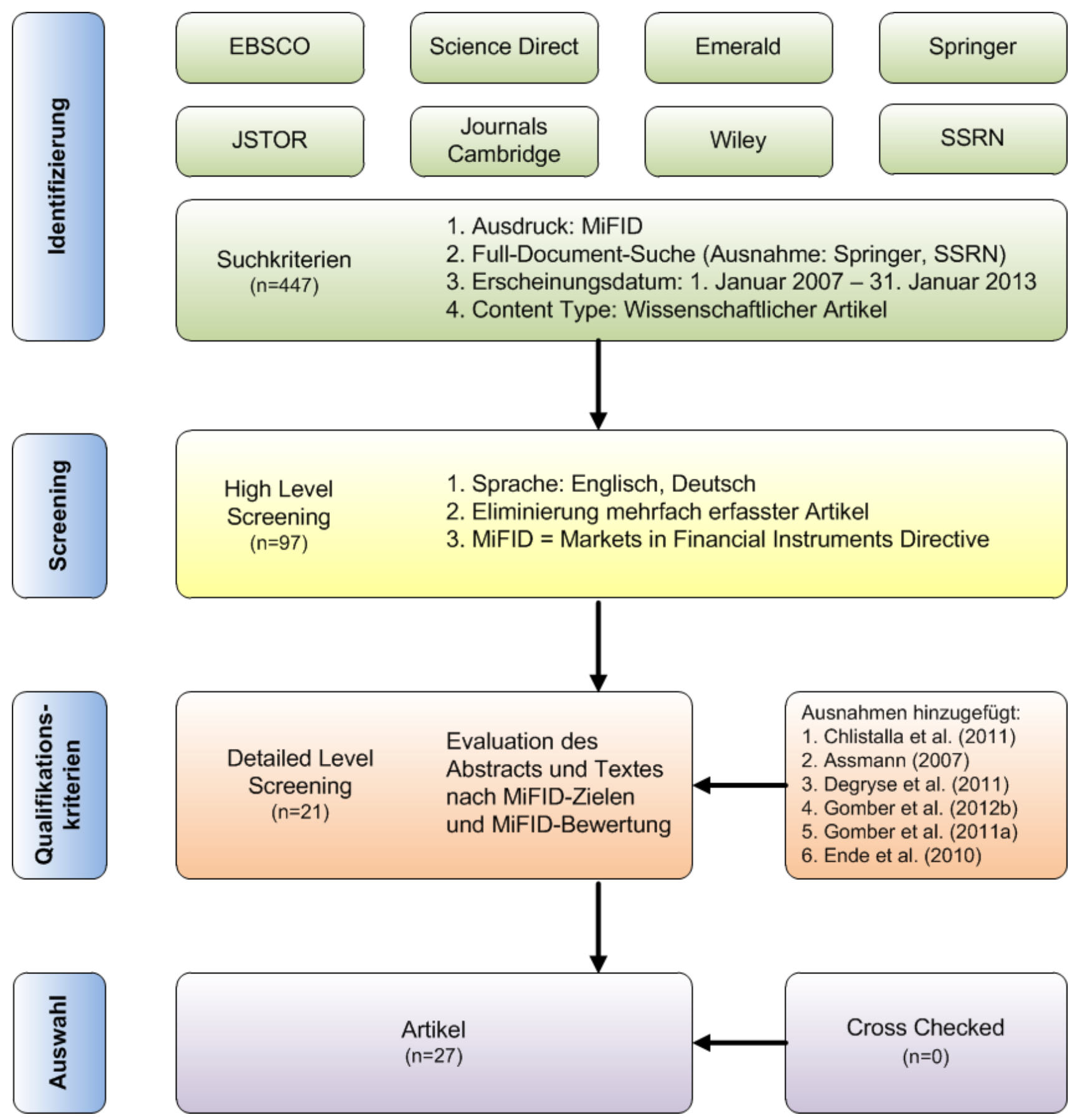

Abbildung 2: Prozess des systematischen Reviews (Eigene Darstellung in Anlehnung an Moher et al. (2009))

Im Anschluss an die Literaturrecherche wurden die Artikel anhand einer Metaanalyse ausgewertet, die eine aggregierte Sicht der MiFID-Zielerreichung wiedergibt. Tabelle 1 zeigt ne- 
ben der Anzahl an positiven und negativen Bewertungen bezüglich der Erreichung einzelner Zwischenziele auch die in den Artikeln angewendeten Forschungsmethoden auf. ${ }^{67}$

\begin{tabular}{|c|c|c|c|c|c|c|c|c|}
\hline \multirow{3}{*}{$\begin{array}{c}\text { Charakteristika } \\
\text { Bewertung der } \\
\text { Zielerreichung }\end{array}$} & \multicolumn{8}{|c|}{ Kategorie } \\
\hline & \multicolumn{2}{|c|}{ Wettbewerb } & \multicolumn{2}{|c|}{ Integration } & \multicolumn{2}{|c|}{ Transparenz } & \multicolumn{2}{|c|}{ Integrität } \\
\hline & positiv: 9 & negativ: 2 & positiv: 6 & negativ: 3 & positiv: 1 & negativ: 4 & positiv: 2 & negativ: 8 \\
\hline \multirow{2}{*}{ Forschungsmethode } & \multicolumn{4}{|c|}{ theoretisch } & \multicolumn{4}{|c|}{ empirisch } \\
\hline & \multicolumn{2}{|c|}{ math. Modell: 1} & \multicolumn{2}{|c|}{ Argumentation: 8} & \multicolumn{2}{|c|}{ statistisch: 18} & \multicolumn{2}{|c|}{ qualitativ: 0} \\
\hline
\end{tabular}

\begin{tabular}{|c|c|c|c|c|c|c|c|c|}
\hline Charakteristika & \multicolumn{8}{|c|}{ Kategorie } \\
\hline \multirow{2}{*}{$\begin{array}{l}\text { 1. Zwischenziel und } \\
\text { Zielerreichung }\end{array}$} & \multicolumn{2}{|c|}{ Wettbewerb } & \multicolumn{2}{|c|}{ Integration } & \multicolumn{2}{|c|}{ Transparenz } & \multicolumn{2}{|c|}{ Integrität } \\
\hline & positiv: 9 & negativ: 2 & positiv: 6 & negativ: 3 & positiv: 1 & negativ: 4 & positiv: 2 & negativ: 8 \\
\hline \multirow{2}{*}{$\begin{array}{l}\text { 2. Forschungs- } \\
\text { methode }\end{array}$} & \multicolumn{4}{|c|}{ theoretisch } & \multicolumn{4}{|c|}{ empirisch } \\
\hline & \multicolumn{2}{|c|}{ math. Modell: 1} & \multicolumn{2}{|c|}{ Argumentation: 8} & \multicolumn{2}{|c|}{ statistisch: 18} & \multicolumn{2}{|c|}{ qualitativ: 0} \\
\hline
\end{tabular}

\section{Tabelle 1: $\quad$ Metaanalyse zu den Ergebnissen des Reviews}

Die Essenz der Einschätzung der Zielerreichung der akademischen Studien spiegelt sich in der Metaanalyse wieder. Wettbewerb und Integration werden bezüglich ihres Beitrages zur Kostensenkung insgesamt sehr positiv bewertet. Kritik zielt nur darauf ab, dass die Möglichkeiten der Kostenreduzierung durch den Wettbewerb und die Integration nicht gänzlich ausgeschöpft wurden. Der Umstand, dass Untersuchungen zu Transparenz, Informationseffizienz und Informationsrisikokosten aufgrund unterschiedlicher Ansätze widersprüchlicher Ergebnisse und konkurrierender Interpretationsmöglichkeiten keine einheitlichen Aussagen liefern ${ }^{68}$, mag ein Grund dafür sein, dass nur wenige Artikel die Transparenzanforderungen bewerten, die Grundtendenzen aber eher negativ sind. Unmissverständlich in ihrer Aussage sind hingegen die Untersuchungen bezüglich der Integrität. Die negative Beurteilung beruht darauf, dass nach Auffassung der Wissenschaftler die Position der Investoren nicht gestärkt wurde. Während sich die Analyse des Wettbewerbs und der Integration statistischer Metho-

\footnotetext{
${ }^{67}$ In der Zählung werden keine Bewertungen berücksichtigt, die innerhalb eines Beitrages sowohl positiv als auch negativ ausfallen. Da mehrere Artikel zu verschiedenen Zwischenzielen Aussagen treffen, entstehen Doppelzählungen in Tabelle 1.

${ }^{68}$ Vgl. Bienert (1996); vgl. Porter et al. (1998); vgl. Boehmer et al. (2005); vgl. Hendershott et al. (2005); vgl. Madhavan et al. (2005); vgl. Chung et al. (2009).
} 
den bediente, basieren die Untersuchungen zur Integrität vor allem auf theoretischen Argumentationen.

\subsection{Bewertung der MiFID-Zielerreichung anhand der wissenschaftlichen Literatur}

Die untersuchten Artikel gliedern sich in drei Gruppen. Die erste Gruppe fasst Artikel zusammen, die eine Veränderung der impliziten Handelskosten auf den Wettbewerb und die technische Integration zurückführen. Die technische Integration wird hierbei als eine Voraussetzung für mehr Wettbewerb betrachtet, denn Wettbewerb zwischen den Marktplätzen entsteht nur, wenn Marktteilnehmer auf verschiedene Handelssysteme zugreifen können. Die zweite Gruppe fasst die Studien zusammen, die den Aktienhandel in erster Linie in Bezug auf Transparenz und den verdeckten Handel in Dark Pools betrachten. Die Studien zeigen Kosten auf, die im Fall vollständiger Informationen aller Marktteilnehmer nicht aufgetreten wären, bzw. Kosten, die einigen Marktteilnehmern entstehen, weil sie nur die Liquidität transparenter Märkte nutzen. In der dritten Gruppe werden schließlich Artikel gebündelt, die sich dem Investorenschutz widmen. Diesen Studien liegt meist die Annahme zugrunde, dass erst die Stärkung der Rechtsposition des Anlegers diesen veranlasst, am Markt tätig zu werden. Maßnahmen zur Wahrung der Integrität sollen Handelskosten reduzieren, indem ökonomisch sinnvolle Transaktionen ausgeführt werden, die ohne Regulierung unterbleiben würden.

\subsubsection{Wettbewerb und Integration}

Die positive Wirkung des Wettbewerbs auf implizite Handelskosten in europäischen Staaten, in denen es in der Vergangenheit keine Konzentrationsregel gab, wird bereits für die PreMiFID Periode aufgezeigt. ${ }^{69}$ Vor diesem Hintergrund leitet Degryse (2009) ab, dass der durch MiFID geschaffene verschärfte Wettbewerb die Liquidität verbessern wird.

Gomber et al. (2011a) untermauern die positive Entwicklung durch Wettbewerb und Integration. Die Autoren vergleichen (in einem Pre-MiFID versus Post-MiFID Beobachtungszeitraum) die Veränderungen der Liquidität (Spread, Marktbreite und Markttiefe) fragmentier-

\footnotetext{
${ }^{69}$ Vgl. Foucault et al. (2008). Für die USA zeigt Hamilton (1979) bereits in den 1970er Jahren, dass Wettbewerb zwischen Handelsplätzen den Spread am Heimatmarkt zu reduzieren vermag.
} 
ter europäischer Blue-Chips mit spanischen Blue-Chips, deren Handel aufgrund der Handelsabwicklung nach wie vor auf den Heimatmarkt konzentriert ist. Während die Liquidität europäischer Blue-Chips unter Wettbewerbsbedingungen sowohl am Heimatmarkt als auch in einem virtuell konsolidierten Orderbuch deutlich zunimmt, ist im Handel spanischer Aktien ein Rückgang der Liquidität zu verzeichnen.

Gresse (2012) liefert einen weiteren Nachweis, dass Wettbewerb und Integration die Liquidität verbessern. Nach Eintritt der MTFs Chi-X, BATS Europe, Turquoise und PLUS in den Handel britischer Blue-Chips (FTSE 100), französischer Blue-Chips (CAC 40) und französischer Mid-Caps (SBF 120 abzüglich CAC 40) verkleinert sich der quotierte und effektive Spread sowohl in einem virtuell konsolidierten Orderbuch als auch auf den jeweiligen geregelten Märkten. Tendenziell verkleinert sich der Spread für häufig gehandelte und LSE-gelistete Aktien am meisten, was auf einen verschärften Wettbewerb für diese Papiere zurückgeführt wird. Soltani et al. (2011) bestätigen die Reduzierung des quotierten Spread für die Aktien des Euronext 100.

Weitere Studien beweisen die positive Wirkung von Wettbewerb und Integration auf die impliziten Handelskosten. Riordan et al. (2011) zeigen, dass die Liquidität (quotierter und effektiver Spread) für Aktien des FTSE $100 \mathrm{im}$ Zeitverlauf sowohl am etablierten Markt LSE als auch an den MTFs Chi-X, BATS Europe und Turquoise steigt. Die Messung der Reduzierung des quotierten und effektiven Spreads sowohl an der LSE als auch an den MTFs Chi-X, BATS Europe und Turquoise wird von Spankowski et al. (2012) bestätigt.

Degryse et al. (2011) stützen diese Erkenntnisse nur teilweise. Die Liquidität (Spread, Marktbreite und Markttiefe) niederländischer Blue-Chips nimmt in einem virtuell konsolidierten Orderbuch $\mathrm{zu}^{70}$, am Heimatmarkt Euronext Amsterdam jedoch ab. Marktteilnehmer, die nicht mit SOR Technologien ausgestattet sind, finden sich durch die Fragmentierung in einer verschlechterten Situation wieder. Der Markt für niederländische Aktien ist somit nicht für alle Marktteilnehmer vollständig integriert.

Hengelbrock et al. (2009) untersuchen den Eintritt des MTFs Turquoise in den Handel von Aktien 14 europäischer Staaten im Jahr 2008. Die Ergebnisse dieser Untersuchung sind weni-

\footnotetext{
${ }^{70}$ Interessant ist, dass sich die Liquidität im konsolidierten Orderbuch nahe des Midpoints weitaus mehr verbessert als die Liquidität nachplatzierter Quotierungen (Markttiefe).
} 
ger eindeutig, tendieren aber insgesamt in eine positive Richtung. Die Autoren messen bezogen auf den MTF-Handel eine Verkleinerung des Spreads für Wertpapiere mit einem verhältnismäßig hohen Spread im Heimatmarkt, ansonsten aber einen erweiterten Spread für Turquoise gegenüber dem Heimatmarkt. ${ }^{71}$ Ferner stellen sie insgesamt keine Zunahme des Handelsvolumens und nur eine mögliche kleine Reduzierung des Spreads im Heimatmarkt fest.

Der Fokus des Ergebnisses der Untersuchung von Chlistalla et al. (2011) richtet sich nicht auf die Kontrastierung der Liquidität verschiedener Marktplätze sondern auf die Gegenüberstellung der Liquidität verschiedener Aktien. Die Autoren analysieren die Wirkung des Handelseintritts des MTFs Chi-X auf die Liquidität am Heimatmarkt Euronext Paris für französische Blue-Chips und konstatieren, dass sich anhand des Wettbewerbs der quotierte Spread am Heimatmarkt für Aktien mit einem hohen Handelsvolumen verengt, wohingegen sich die Situation für weniger häufig gehandelte Aktien nicht verbessert. Das Auftauchen eines neuen Mitbewerbers hat demnach keinen positiven Einfluss auf die Liquidität am etablierten Markt, wenn die Handelsaktivität verhältnismäßig niedrig ist. Gresse (2012) und Soltani et al. (2011) liefern in diesem Zusammenhang identische Ergebnisse. Eine Ausnahme der positiven Entwicklung der Liquidität bilden auch in ihren Analysen die weniger fragmentierten französischen Mid-Caps bzw. die Mid-Caps und Small-Caps des Euronext 100-250, bei denen sich jeweils der Spread nicht verbessert.

Ribeiro (2010) stellt die Frage, was den Handel der 16 FTSE 100 Aktien mit dem größten Handelsvolumen kurz nach der Einführung der MiFID davon abhält sich am Handelsplatz zu konzentrieren, der die geringsten expliziten Handelskosten aufweist. Nach der Auffassung des Autors wird der Wettbewerb durch Netzwerkeffekte und Skaleneffekte sowie durch die monopolähnliche Handelsabwicklung beschränkt, wovon etablierte Börsen profitieren. Mit Hilfe eines Modells stellt er den Handel nach, erstens, als gäbe es keine Liquiditätsunterschiede zwischen den Ausführungsorten (einheitlicher effektiver Spread) und, zweitens, als wäre die Dienstleistung der Handelsabwicklung fungibel. In beiden Simulationen verschieben sich die Marktanteile zum Handelsplatz mit den geringsten expliziten Kosten (MTFs und SIs).

\footnotetext{
${ }^{71}$ Das Ergebnis gilt für den quotierten und effektiven Spread gleichermaßen. Auf fast allen Märkten ist der effektive Spread geringer als der quotierte Spread, was darauf zurückzuführen ist, dass mehr Transaktionen zu Zeiten eines engen Spreads ausgeführt werden, und somit der Durchschnitt des quotierten Spreads den Durchschnitt des effektiven Spreads übersteigt.
} 
Die freie Wahl des Dienstleitungsanbieters nicht nur bezüglich der Orderausführung, sondern auch in Bezug auf die Handelsabwicklung, würde die Handelskosten im Wertpapierhandel weiter reduzieren. Gentile et al. (2011) sehen ebenfalls einige etablierte Börsen aufgrund von ihrer ehemaligen Monopolstellung über Netzwerkeffekte des Wertpapierhandels im Vorteil.

Storkenmaier et al. (2011) zeigen, dass Quotierungen für britische Blue-Chips (FTSE 100) über Marktplätze (LSE, Chi-X, BATS, Turquoise) hinweg eng aneinander gekoppelt sind, und dass ein hoher Anteil des Handels zu den bestverfügbaren Preisen ausgeführt wird. ${ }^{72}$ Ein Marktplatz zeigt ein besonders hohes Handelsvolumen in Zeiten in denen er alleine das europaweite Best Bid Offer (EBBO) stellt. Die Anzahl an Trade-Throughs nimmt im Zeitverlauf ab. Die Tickgröße des „negativen“ Spread sowie der Zeitraum, in dem das European Best Bid (EBB) dem European Best Offer (EBO) gleicht (locked Quotierungen), bzw. das EBB das EBO überschreitet (crossed Quotierungen), nimmt im Zeitverlauf rapide ab. ${ }^{73}$ Der Wettbewerb und die genaue Beobachtung der Marktsituation durch die Marktteilnehmer zwingen konkurrierende fragmentierte Marktplätze Quotierungen zu stellen, als wären sie formal verknüpft.

Ende et al. (2010) betrachten die Effizienz der Orderausführung europäischer Blue-Chips (Euro Stoxx 50) kurz nach Einführung der MiFID, indem sie die Ausführung auf zehn kontinentaleuropäischen Marktplätzen ${ }^{74}$ mit Hilfe der SOR Technologie nachstellen. Der SORAlgorithmus durchsucht das beste Bid und Ask der Märkte und zeigt selbst unter Einbezug der variablen expliziten Kosten (inklusive Kosten für das Clearing und Settlement) Potenzial für Kosteneinsparungen auf. Der Gesetzgeber könnte anhand eines strikten Trade-ThroughVerbotes die Kosten des Wertpapierhandels für Anleger weiter senken.

\footnotetext{
72 Während im Untersuchungszeitraum 2009 die LSE den engsten quotierten Spread und am häufigsten die besten verfügbaren Preise stellt, übernimmt Chi-X diese Position im Untersuchungszeitraum 2010. Im Zeitverlauf verkleinert sich der quotierte Spread auf allen Marktplätzen. Die LSE weist nach wie vor die größte Marktbreite auf.

73 Insgesamt treten Trade-Throughs viel seltener auf als dies von Foucault et al. (2008) für das Jahr 2004 gemessen wurde. Die locked und crossed Quotierungen sind jedoch mit einem effizienten Markt nicht vereinbar. Die locked Quotierung kennzeichnet eine Situation in der es in einem konsolidierten Orderbuch zu einem Handel käme. Die crossed Quotierung bietet die Möglichkeit der Arbitrage und verletzt das Law-of-one-Price.

${ }^{74}$ Bolsa de Madrid, Borsa Italiana Milan, Chi-X, Euronext Amsterdam/ Brüssel/ Paris/ Lisabon, NASDAQ OMX Helsinki, SWX Europe und Xetra.
} 
Insgesamt zeigen die Studien, dass sich die konsolidierte Liquidität auf den Marktplätzen gegenüber der Pre-MiFID Zeit verbessert hat. Den mit Kosten verbundenen Zugriff in Echtzeit auf verschiedene Marktplätze müssen Marktteilnehmer jedoch selbst organisieren.

\subsubsection{Transparenz}

Degryse et al. (2011) zeigen empirisch auf, dass sich der Handel auf Plattformen ohne Vorhandelstransparenz negativ auf die Liquidität (Spread, Marktbreite, Markttiefe) niederländischer Blue-Cips und Mid-Caps auswirkt. Der Wettbewerb bzw. die Fragmentierung kann die konsolidierte Liquidität gegenüber der Liquidität eines einzigen zentrierten Marktplatzes insgesamt bis zu 30 Prozent verbessern, weil sich der Wettbewerb unter den Anbietern von Liquidität erhöht. Der verdeckte Handel hingegen reduziert die Liquidität sowohl im konsolidierten Orderbuch als auch im Heimatmarkt, weil Limit-Aufträge in die Dark Pools migrieren.

Gomber et al. (2010) untersuchen Transaktionen, die auf OTC-Basis abgeschlossen werden, und überprüfen die Tradegrößen der OTC-Transaktionen relativ zur standardmäßigen Marktgröße (SMS). Weiterhin wird hier geprüft, ob OTC-Transaktionen, wenn sie auf dem Markt mit der größten Liquidität ausgeführt worden wären, eine Marktwirkung gehabt hätten. Die Studie stellt fest, dass die Volumina der Hälfte der Transaktionen der Euro Stoxx 50 Aktien nicht über der SMS (zumeist bei 7.500€) liegen, wobei dieser Anteil im Zeitverlauf von durchschnittlich 41\% (2008) auf 54\% (2010) steigt, hingegen der Anteil der Large-in-ScaleTransaktionen (LIS; $\geq 500.000 €$ ) von durchschnittlich 15\% (2008) auf 9\% (2010) fällt. Ferner hätten durchschnittlich 68\% im Jahr 2008 und 80\% im Jahr 2010 der OTC-Transaktionen keine (negative) Marktwirkung im offenen Orderbuch des führenden Marktes gehabt. ${ }^{75}$

Wenige Artikel befassen sich mit dem Informationsgehalt von Aufträgen. Riordan et al. (2011) zeigen, dass Chi-X unter der Berücksichtigung von Preisen und Quotierungen zunehmend mehr zur Preisfindung beiträgt als der Heimatmarkt LSE, was auf dem hohen Informationsgehalt der Quotierungen beruht. Demnach kann die Behauptung des Free-Riding von MTFs bezüglich der Preisfindung widerlegt werden. Nach der Studie von Spankowski et al. (2012) vertrauen Investoren hingegen zumindest zum Handelsauftakt nach wie vor auf die

\footnotetext{
${ }^{75}$ Mit wenig liquiden Aktien verhält es sich ähnlich. Die Volumina der Transaktionen die unter der SMS liegen, steigen im Zeitverlauf von durchschnittlich 54\% (2008) auf 61\% (2010). Hingegen fällt der Anteil der LISTransaktionen von durchschnittlich 6\% (2008) auf 4\% (2010). Ferner hätten hier im Durchschnitt im Jahr 2008 58\% und im Jahr 2010 66\% der OTC-Transaktionen keine (negative) Marktwirkung gehabt.
} 
LSE als dem Ort, der den größten Informationsgehalt zur Preisfindung beiträgt ${ }^{76}$ und zu diesem Zeitpunkt das mit Abstand größte Handelsvolumen aufweist, woraus sich bezogen auf das Handelsvolumen für die LSE eine U-Form ${ }^{77}$ und für die MTFs ein J-Form abbilden lässt.

Gentile et al. (2011) liefern den einzigen Beitrag, der die Informationseffizienz der Pre-MiFID Zeit mit der fragmentierten Marktsituation der Post-MiFID Periode vergleicht. Zwar weist der Handel fragmentierter Aktien (FTSE) gegenüber nicht-fragmentierten Aktien (IBEX) eine Verbesserung der Liquidität (quotierter Spread) auf, die Informationseffizienz wird von der Fragmentierung hingegen negativ beeinflusst. ${ }^{78}$ Als Ursache nennen die Autoren die mangelhafte Konsolidierung der Vor- und Nachhandelsdaten und fordern daher die Einführung eines CTS.

Nach Petrella (2010) verpflichtet die MiFID zwar die Märkte Informationen wie den Preis, das Handelsvolumen und den Handelszeitpunkt zu veröffentlichen, eine Datenkonsolidierung wird jedoch nicht vorgeschrieben. Dies hat eine Verschlechterung der Preisfindung zur Folge. Außerdem versäumt es die MiFID, sowohl den Marktplätzen als auch den Intermediären die Veröffentlichung von Statistiken bezüglich der Qualität der Ausführung zwingend vorzuschreiben. ${ }^{79}$ Von den beschriebenen Defiziten profitieren die ehemaligen Börsenmonopole und Intermediäre.

Degryse et al. (2011) unterscheiden drei Typen von Anlegern mit unterschiedlichem Zugriff auf Informationen. Eine Gruppe von Händlern durchkämmt alle Märkte, generiert ein virtuell konsolidiertes Orderbuch und teilt Orders entsprechend auf. Eine zweite Gruppe wählt den Handelsort, der aktuell die besten Handelsmöglichkeiten bietet, kann aber nicht simultan auf mehrere Plattformen zugreifen. Eine dritte Gruppe handelt wie bisher nur am Heimatmarkt. Während die erste Gruppe allgemein von der Fragmentierung profitiert, verbessert sich die Situation der zweiten Gruppe nur für kleine Aufträge. Die dritte Gruppe hingegen sieht sich mit gestiegenen impliziten Handelskosten konfrontiert. Trotz dieser gravierenden Unter-

\footnotetext{
${ }^{76}$ Nach Glosten et al. (1985) weitet sich der quotierte Spread aus, wenn der Anteil informierter Handelsaufträge steigt.

${ }^{77}$ Eine U-Form bildet sich, weil zu Handelsbeginn Portfolios aufgrund der Informationen über Nacht angepasst werden müssen und Marktteilnehmer Positionen zu Handelsende ausgleichen. Vgl. Brock et al. (1992).

${ }^{78}$ Aufgrund der genannten Schwierigkeiten bei der Analyse der Informationseffizienz reicht eine einzelne Stellungnahme zu einer soliden Aussage bezüglich der Veränderung der Informationseffizienz durch die MiFID nicht aus.

${ }^{79}$ Analog der Reg NMS könnten Marktplätze über die Wahrscheinlichkeit sowie Geschwindigkeit der Ausführung und die Preisverbesserung berichten bzw. Intermediäre über die Orte der Ausführung.
} 
schiede in den Ergebnissen erfüllt jede dieser Handelsstrategien die MiFID-Anforderungen der Best Execution, die im folgenden Unterkapitel analysiert wird.

\subsubsection{Integrität}

Cumming et al. (2011a \& 2011b) heben die Bedeutung von Handelsregeln zum Schutz der Anleger hervor. Die Verfasser untersuchen wie Handelsregeln die Wahl eines Handelsplatzes bei mehrfach gelisteten Aktien beeinflussen und finden heraus, dass das Handelsvolumen von MiFID-Marktplätzen wächst, indem sich der Handel mehrfach gelisteter Aktien von U.S. amerikanischen Marktplätzen auf Handelsplätze innerhalb der EU verlagert. Die Wirkung zum Zeitpunkt der Verabschiedung der MiFID war dabei weitaus positiver als der Effekt der tatsächlichen Einführung. Zudem wird der geringere Spread innerhalb der EU in der PostMiFID-Periode gegenüber der Pre-MiFID-Periode auf die Einführung einheitlicher Handelsregeln zurückgeführt.

Gomber et al. (2012b) vergleichen die Best Execution Policies der größten deutschen Banken und der Online-Broker des Jahres 2008 und 2009. Der Inhalt der Policies wurde trotz der sich stark verändernden Wettbewerbsbedingungen und der Fragmentierung der Liquidität in mehr als der Hälfte der Fälle von den Instituten auch nach über einem Jahr nicht überarbeitet. Bei einer Mehrheit der Best Execution Policies (73\%) fehlt die konkrete Angabe oder Rangfolge der favorisierten Marktplätze. Ferner erwähnt nur ein einziger Intermediär den Einsatz von Echtzeitdaten und modernen Lenkungstechniken wie dem SOR. Deutsche Broker nutzen nach wie vor inländische Börsen. MTFs werden hingegen in keiner einzigen Policy genannt. Die Best Execution wird nicht als Differenzierungsmerkmal im Konkurrenzkampf genutzt, sondern als eine regulatorische Notwendigkeit betrachtet.

Ferrarini (2007) sieht in der Priorisierung des Gesamtentgeltes auf der zweiten Ebene und dem damit verbundenen Einbezug der Kosten für die Handelsabwicklung eine Barriere für alternative Handelsmechanismen. Entgegen dem ursprünglichen Zweck der MiFID profitieren etablierte Börsen von diesen, den Wettbewerb einschränkenden Best Execution Maßnahmen, weil sie aufgrund ihrer angeschlossenen günstigeren Möglichkeiten der Handelsabwicklung und ihrer historisch bedingten hohen Liquidität auf lange Sicht die Kriterien der statischen Best Execution besser erfüllen. In diesem Zusammenhang bemängelt auch Petrella (2010), dass die statische Best Execution Policy die Integration der Märkte verhindert und für den Anleger die Beurteilung der Qualität der Ausführung erschwert. 
Assmann (2007) prüft die MiFID auf Interessenskonflikte in der Anlageberatung. Hierzu betrachtet er Geldleistungen oder geldwerte Vorteile, die Intermediäre im Zusammenhang mit der Erbringung von Wertpapierdienstleistungen für den Kunden annehmen oder Dritten gewähren (Inducements). ${ }^{80}$ Problematisch an Inducements ist, dass diese Anreize setzen können, die dem Kundeninteresse zuwiderlaufen und, dass die Ausnahme des InducementsVerbots gültig ist, wenn die Zuwendung des Dritten an einen Beauftragten des Kunden gezahlt wird oder von einem Beauftragten des Kunden an einen Dritten gewährt wird. Dies ist nicht nachvollziehbar, weil eine Wertpapierfirma gerade mittels der Beauftragung durch den Kunden in einen Interessenskonflikt geraten kann. Zudem ist die Annahme von Provisionen nach der MiFID-Durchführungsrichtlinie im Rahmen der Anlageberatung gerechtfertigt, wenn „[sie] eine qualitative Verbesserung der Anlageberatung gegenüber dem Kunden bezweckt. ${ }^{\prime 81}$. Dies bedeutet, dass der Anleger nachweisen muss, dass die Anlageberatung voreingenommen erfolgte und die Qualität der Beratung offenkundig fehlerhaft war. Insgesamt bewertet Assmann die MiFID-Regelung des Broker-Anleger Konfliktes als eine „widersprüchliche, inkonsistente sowie in großen Teilen kompromisszersetzte und zu allem Überfluss noch hyperkomplexe und hyperkomplizierte Regelungsvorgabe ${ }^{\prime \prime 2}$.

Inderst (2009) beschränkt sich in seiner Forderung zur Verbesserung des Anlegerschutzes auf ein erweitertes Rücktrittsrecht. Die Offenlegung von verdeckten Provisionen, die Produktanbieter an Intermediäre zahlen, kann der Autor anhand theoretischer Modelle nicht pauschal befürworten. Während Transparenz insbesondere bei leichtgläubigen Anlegern den Interessenskonflikt schmälert, unterbindet sie gleichzeitig den Anreiz für Intermediäre sich Informationen für die Beratung zu beschaffen und neue, effizientere Produkte am Markt zu platzieren. ${ }^{83}$ Die Regulierung sollte diesen Aspekt bei einer Offenlegungspflicht oder Deckelung der Provisionen berücksichtigen.

Cherednychenko (2010) untersucht, in welchem Umfang die MiFID Privatinvestoren befähigt, ihre Rechte durchzusetzen. Der Anspruch der MiFID, jedem Anleger das gleiche Maß an Investorenschutz zuzugestehen, wird nach Einschätzung des Autors nicht erfüllt. Die MiFID gewährt Investoren nicht die Möglichkeit ihr Recht aktiv und direkt gegenüber pflichtverlet-

\footnotetext{
${ }^{80}$ Vgl. Assmann (2007).

${ }^{81}$ Europäische Kommission (2006a) Erwägungsgrund 39.

${ }^{82}$ Assmann (2007).

${ }^{83}$ Für eine Betrachtung der dieser Argumentation zugrunde liegenden Modelle siehe Inderst et al. (2009).
} 
zenden Intermediären einzufordern, sondern überlässt dies der privatrechtlichen Durchsetzung der Nationalstaaten, die die aufsichtsrechtlichen Standards unterschiedlich anwenden. Zudem stellen nur wenige Staaten kollektive Rechtsdurchsetzungsverfahren bereit. Ferner verpasst es die MiFID, die Nachweispflichten in Rechtsstreitigkeiten dem Dienstleistungsanbieter aufzubürden, wie dies in anderen Direktiven der Fall ist. Die MiFID stärkt weder die Rechtsposition des Anlegers, noch schafft sie gleiche Bedingungen für Investoren in Europa ihre Rechte durchzusetzen.

In derselben Weise beanstandet Burke (2009), dass die MiFID es den Nationalstaaten überlässt, wie Investoren ihr Recht geltend machen können. Ferner liefert sie keine Grundlage für Sammelklagen, die nach Auffassung des Autors eine abschreckende Wirkung hätten, Investoren systematisch zu schädigen. Trotz der schlechten Statistiken in der Aufklärung von Missbrauchsfällen im Wertpapierhandel beinhaltet die MiFID im Gegensatz zur Regulierung in den USA keine Sanktionsmaßnahmen wie Bußgelder und Freiheitsstrafen.

Steennot (2008) kritisiert gleichermaßen, dass die MiFID kaum Sanktionsmöglichkeiten bietet. Im Fall einer Missachtung der Wohlverhaltensregeln durch den Broker kann auf Grundlage von Artikel 27 der Durchführungsrichtlinie die an ihn im Rahmen der Leistungserbringung gezahlte Vergütung zurückgefordert werden. Die aus einer Falschberatung resultierenden Investitionsverluste lassen sich hingegen nur im nationalen zivilrechtlichen Verfahren erstreiten, wobei dem Anleger die Beweislast obliegt. Zudem vertritt Burke (2009) die Auffassung, dass der durchschnittliche Privatinvestor mit der Masse an Informationen überfordert ist und diese daher nicht beachten wird. Deshalb sollten essentielle Informationen wie Transaktionskosten und Risiken dem Kunden getrennt von weiterführenden Informationen übergeben werden. Klöhn (2009) argumentiert auf der Basis theoretischer und empirischer Befunde der Behavioural Finance und fordert, dass Intermediäre das Handelsverhalten von Discount-Brokerage-Kunden beobachten und sie bereits im Vorfeld einer Investitionsentscheidung aktiv, individuell und situationsspezifisch vor Risiken warnen.

Gomber et al. (2012a) untersuchen die Beziehung zwischen systematischer Internalisierung und Best Execution. Die Verfasser weisen empirisch nach, dass in 92\% der Fälle die Internalisierung für Investoren vorteilhaft oder zumindest nicht mit Nachteilen verbunden ist und dass wenige große Handelstransaktionen (>60.000€) die Kosten für die Preisverbesserungen vieler kleiner Orders $(\leq 60.000 €)$ tragen, was möglicherweise auf das für den SI höhere Risiko 
großer Aufträge zurückzuführen ist. Momentan ist das Risiko, die Internalisierung könne die Geld-Brief-Spanne auf den Referenzmärkten ausweiten, aufgrund der spärlichen Anzahl an SIs und deren vergleichsweise niedrigem Handelsvolumen gering. ${ }^{84}$ Die Studie von Gresse (2012) bezüglich der Wirkung der Internalisierung auf die Liquidität von an der LSE und Euronext gehandelten Aktien kommt ebenfalls zu keinem negativen Ergebnis. Einer möglichen Verbesserung der Marktbreite steht eine mögliche Verschlechterung des quotierten Spread gegenüber.

\section{Zusammenfassung und Ausblick}

Ziel der MiFID ist es, den Wertpapierhandel effizient zu gestalten, damit die Kosten für Investoren sinken. Hierzu beschloss die Europäische Kommission den Wettbewerb, die Integration, die Transparenz und die Integrität zu fördern. Auf der operativen Ebene dienen Wettbewerb und Integration der Reduktion von Handelskosten. Die korrekte Kalibrierung der Transparenz schützt Anleger gegen Informationsrisikokosten. Aufgabe der Integrität ist es, die Bereitschaft zu ökonomisch sinnvollem Handel zu erhöhen.

Die Mehrzahl der hier analysierten akademischen Studien adressiert die gemeinsame Wirkung von Wettbewerb und Integration auf die Liquidität. Die Bewertung der Wissenschaftler ist positiv, weil explizite und zumeist auch implizite Handelskosten sinken. Die Europäische Kommission erreicht es mit ihren Maßnahmen jedoch nicht, die Liquidität für alle Marktteilnehmer zu verbessern. Zudem wird die auf Basis eines CQS ermittelte Ausführung zum EBBO in Verbindung mit einem Trade-Through-Verbot nicht verlangt. Die genannten Funktionalitäten werden zwar bereits von professionellen Marktteilnehmern anhand von SOR-Systemen dezentral eingesetzt; Privatanleger haben hingegen durch ihre eingeschränkten Zugriffsmöglichkeiten keine Garantie einen besten Preis zu erzielen. Von der durch die MiFID erzielten Kostensenkung profitieren in erster Linie die Intermediäre. Darüber hinaus ist die Forderung nach einer einheitlichen Transparenzregelung für alle Mechanismen der Orderausführung ${ }^{85}$ unzureichend umgesetzt, weil sich Börsen und MTFs der Regulierung unterziehen müssen, sich funktional identische Teile des OTC-Handels aber einer Kontrolle entziehen können.

\footnotetext{
84 Momentan sind 13 Sls registriert von denen die meisten in GB ansässig sind. Vgl. http://mifiddatabase.esma.europa.eu/Index.aspx?sectionlinks_id=16\&language=0\&pageName=MiFIDSystem aticSearch\&subsection_id=0 (Zugriff: 15.11.2012). Das Volumen beträgt 2 Prozent des gesamten europäischen Aktienhandels. Vgl. Gomber et al. (2012a).

${ }^{85}$ Europäisches Parlament (2004) Erwägungsgrund 44.
} 
Schließlich wird die Erreichung des Zwischenziels der Integrität als gescheitert beurteilt. Der Anleger genießt weder in der Beratung gegenüber dem Intermediär einen höheren Schutz noch wurde seine Situation bezüglich der Orderausführung anhand der Best Execution Policy, die in der Realität eine Litanei des Gesetzestextes bleibt, gegenüber der Vergangenheit verbessert. Werden Anleger von Intermediären erwiesenermaßen geschädigt, so schützt die MiFID sie nicht. Anleger sind stattdessen auf die verschiedenen zivilrechtlichen Klageverfahren ihrer Nationalstaaten angewiesen. Ferner wird die Veröffentlichung von Indikatoren über die Qualität der Ausführung nicht vorgeschrieben. ${ }^{86}$

Die Symbiose aus Wettbewerb, Integration, der korrekten Dosis an Transparenz und Integrität, sind die Komponenten, die zu einem ökonomisch vorteilhaften, einheitlichen europäischen Wertpapiermarkt führen können. Dieses Potential und die damit für den Anleger verbundenen Kosteneinsparungen wurden von der MiFID nur partiell erreicht und werden in der Neufassung der MiFID (MiFID 2) lediglich teilweise weiterverfolgt. Diese Neufassung der MiFID hat sich als Folge der Finanzkrise primär zur Förderung der Stabilität und Sicherheit anstelle der Effizienz und Kostensenkung verpflichtet. ${ }^{87}$

\footnotetext{
${ }^{86}$ Boehmer et al. (2007) haben für die USA empirisch nachgewiesen, dass Marktplätze, die geringe Handelskosten und schnelle Ausführungszeiten berichten, in den folgenden Monaten ein höheres Auftragsvolumen aufweisen.

${ }^{87}$ Vgl. Europäische Kommission (2011).
} 


\section{Literaturverzeichnis}

Akerlof, G. und Shiller, R. (2009): “Animal Spirits: wie Wirtschaft wirklich funktioniert", Campus Verlag.

Akerlof, G. (1970): “The Market for 'Lemons': Quality Uncertainty and the Market Mechanism", in: Quarterly Journal of Economics, Vol. 84, Nr. 3, S. 488-500.

Amihud, Y. und Mendelson, H. (2006): "Stock and bond liquidity and its effect on prices and financial policies", in: Financial Markets and Portfolio Management, Vol. 20, Nr. 1, S. 19-32.

Amihud, Y. und Mendelson, H. (1986): "Asset Pricing and the Bid-Ask Spread", in: The Journal of Financial Economics, Vol. 17, S. 223-249.

Assmann, H.-D. (2007): “Interessenkonflikte und „Inducements“ im Lichte der Richtlinie über Märkte für Finanzinstrumente (MiFID) und der MiFID-Durchführungsrichtlinie", in: BankArchiv: Zeitschrift für das gesamte Bank- und Börsenwesen, Vol. 55, Nr. 1, S. 40-55.

Baumol, W. (1982): "Contestable Markets: An Uprising in the Theory of Industry Structure", in: The American Economic Review, Vol. 72, Nr. 1, S. 1-15.

Beckmann, K. (1998): “Analytische Grundlagen einer Finanzverfassung: Regelbegründungen und Regelbindungsprobleme aus finanzwissenschaftlicher Perspektive", Lang.

Bienert, H. (1996): “Der Marktprozess an Aktienbörsen: Bewertungseffizienz und Umverteilung", Gabler.

Boehmer, E., Jennings, R. und Wei, L. (2007): "Public disclosure and private decisions: equity market execution quality and order routing", in: Review of Financial Studies, Vol. 20, Nr. 2, S. 315-58.

Boehmer, E., Saar, G. und Yu, L. (2005): "Lifting the Veil: An Analysis of Pre-trade Transparency at the NYSE", in: The Journal of Finance, Vol. 60, Nr. 2, S. 783-815.

Brock, W. und Kleidon, A. (1992): "Periodic market closure and trading volume: A model of intraday bids and asks", in: Journal of Economic Dynamics and Control, Vol. 16, Nr. 3-4, S. 451-489.

Burke, J. (2009): "Investor Protection under MiFID: Cure Worse than the Disease", Working Paper.

http://papers.ssrn.com/sol3/papers.cfm?abstract_id=1329871 (Zugriff: 31.01.13).

Butler, A., Grullon, G. und Weston J. (2005): "Stock Market Liquidity and the Cost of Issuing Equity", in: The Journal of Financial Quantitative Analysis, Vol. 40, Nr. 2, S. 331-348.

Cherednychenko, O. (2010): "The Regulation of Retail Investment Services in the EU: Towards the Improvement of Investor Rights?", in: Journal of Consumer Policy, Vol. 33, Nr. 4, S. 403-424.

Chlistalla, M. und Lutat, M. (2011): "Competition in Securities Markets: The Impact on Liquidity", in: Financial Markets and Portfolio Management, Vol. 25, Nr. 2, S. 149-172.

Chowdhry, B. und Nanda, V. (1991): "Multimarket trading and market liquidity", in: Review of Financial Studies, Vol. 4, S. 483-511. 
Chung, K. und Chuwonganant, C. (2009): "Transparency and Market Quality: Evidence from SuperMontage", in: Journal of Financial Intermediation, Vol. 18, S. 93-111.

Cumming, D., Johan, S. und Li, D. (2011a): "Exchange trading rules and stock market liquidity", in: The Journal of Financial Economics, Vol. 99, Nr. 3, S. 651-671.

Cumming, D., Humphery-Jenner, M. und Wu, E. (2011b): “Exchange Trading Rules, Governance, and Trading Location of Cross-Listed Stocks", 24th Australasian Finance and Banking Conference 2011 Paper; 2012 Financial Markets \& Corporate Governance Conference. http://papers.ssrn.com/sol3/papers.cfm?abstract_id=1789203 (Zugriff: 31.01.2013).

Degryse, H., De Jong, F. und Van Kervel, V. (2011): "The Impact of Dark Trading and Visible Fragmentation on Market Quality", TILEC Discussion Paper Nr. 2011-026. http://papers.ssrn.com/sol3/papers.cfm?abstract_id=1815025 (Zugriff: 31.01.13).

Degryse, H. (2009): "Competition betweerfinancial markets in Europe: what can be e xpected from MiFID?", in: Financial Markets and Portfolio Management, Vol. 23, Nr. 1, S. 93103.

De Haan, J., Oosterloo, S. und Schoenmaker, D. (2009): “European Financial Markets and Institutions", Cambridge University Press.

Dickson, M. (2005): "Nasty Sting", in: Financial Times, 22.07.2005.

http://www.ft.com/intl/cms/s/0/4df1125a-fa4c-11d9-b092-

00000e2511c8.html\#axzz2M7yMXWER (Zugriff: 26.02.2013).

Easley, D. und O'Hara, M. (2009): “Ambiguity and Nonparticipation: The Role of Regulation”, in: Review of Financial Studies, Vol. 22, Nr. 5, S. 1817-1843.

Ende, B. und Lutat, M. (2010): "Trade-throughs in European cross-traded equities after transaction costs-empirical Evidence for the EURO STOXX 50", in: 2nd International Conference: The Industrial Organisation of Securities Markets: Competition, Liquidity and Network Externalities; Frankfurt.

Europäische Kommission (2011): "Vorschlag für eine Richtlinie über Märkte für Finanzinstrumente zur Aufhebung der Richtlinie 2004/39/EG des Europäischen Parlaments und des Rates", 2011/0298 (COD).

Europäische Kommission (2006a): “Richtlinie zur Durchführung der Richtlinie 2004/39/EG", 2006/73/EG (Durchführungsrichtlinie).

Europäische Kommission (2006b): "Markets in Financial Instruments Directive ("MiFID"): implementing measures close to adoption", Pressemitteilung, IP/06/846.

http://europa.eu/rapid/press-release_IP-06-846_en.htm?locale=fr (Zugriff: 26.02.13).

Europäische Kommission (2002a): "Vorschlag für eine Richtlinie über Wertpapierdienstleistungen und geregelte Märkte", 2002/0269 (COD).

Europäische Kommission (2002b): “Wertpapierdienstleistungen: vorgeschlagene neue Richtlinie soll Anleger schützen und EU-weite Tätigkeit von Wertpapierhäusern erleichtern", Pressemitteilung, IP/02/1706.

http://europa.eu/rapid/press-release_IP-02-1706_de.htm (Zugriff: 23.12.12).

Europäisches Parlament und Rat (2004): "Richtlinie über Märkte für Finanzinstrumente", 2004/39/EG (MiFID). 
Fama, E. (1970): "Efficient Capital Markets: A Review of Theory and Empirical Work", in: The Journal of Finance, Vol. 25, Nr. 2, S. 383-417.

Ferrarini, G. (2007): "Best Execution and Competition Between Trading Venues - MiFID's Likely Impact”, in: Capital Markets Law Journal, Vol. 2, Nr. 4, S. 404-413.

Fettke, P. (2006): "State-of-the-Art des State-of-the-Art: Eine Untersuchung der Forschungsmethode "Review" innerhalb der Wirtschaftsinformatik", in: Wirtschaftsinformatik, Vol. 48, Nr. 4, S. 257-266.

Fink, A. (1998): "Conducting research literature reviews: from the Internet to paper", Sage Pubications.

Foucault, T. und Menkveld, A. (2008): "Competition for Order Flow and Smart Order Routing Systems", in: The Journal of Finance, Vol. 63, Nr. 1, S. 119-158.

Friend, I. (1972): "The Economic Consequences of the Stock Market", in: The American Economic Review, Vol. 62, Nr. 1-2, S. 212-219.

Friedman, M. (2002): "Kapitalismus und Freiheit", Eichborn.

Gentile, M. und Fioravanti, S. (2011): "The Impact of Market Fragmentation on European Stock Exchanges", CONSOB Working Paper Nr. 69.

http://papers.ssrn.com/sol3/papers.cfm?abstract_id=1997419 (Zugriff: 31.01.13).

Giannetti, M., Guiso L., Jappelli, T., Padula, M. und Pagano, M. (2002): “Financial market integration, corporate financing and economic growth", European Economy, Economic Papers Nr. 179.

http://ec.europa.eu/economy_finance/publications/publication1660_en.pdf

(Zugriff: 23.02.13).

Glaeser, E., Johnson, S. und Shleifer A. (2001): "Coase Versus the Coasians", in: The Quarterly Journal of Economics, Vol. 116, Nr. 3, S. 853-899.

Glosten, L. und Milgrom, P. (1985): "Bid, Ask and Transaction Prices in a Specialist Market with Heterogeneously Informed Traders", in: The Journal of Financial Economics, Vol. 14, Nr. 1, S. 71-100.

Gomber, P. und Zimmermann, K. (2012a): “Off-Exchange Market Making Price Aggressiveness of Systematic Internalisers", SSRN Working Paper.

http://papers.ssrn.com/sol3/papers.cfm?abstract_id=2173246 (Zugriff: 31.01.13).

Gomber, P., Pujol, G. und Wranik, A. (2012b): "Best Execution Implementation and Broker Policies in Fragmented European Equity Markets", in: International Review of Business Research Papers, Vol. 8, Nr. 2, S. 144-162.

Gomber, P., Gsell, M., Lutat, M. (2011a): “Competition among electronic markets and market quality", in: 14th Conference of the Swiss Society for Financial Market Research (SGF).

Gomber, P. und Pierron, A. (2010): "MiFID - Spirit and Reality of a European Financial Markets Directive", White Paper.

http://papers.ssrn.com/sol3/papers.cfm?abstract_id=1858605 (Zugriff: 31.12.2013).

Gresse, C. (2012): "Effects of Lit and Dark Trading Venue Competition on Liquidity: The MiFID Experience", SSRN Working Paper. 
http://papers.ssrn.com/sol3/papers.cfm?abstract_id=1918473 (Zugriff: 31.01.2013).

Grossman, S. und Stiglitz, J. (1980): “On the Impossibility of Informationally Efficient Markets", in: The American Economic Review, Vol. 70, Nr. 3, S. 393-408.

Hamilton, J. (1979): "Marketplace fragmentation, competition, and the efficiency of the stock exchange", in: The Journal of Finance, Vol. 34, Nr.1, S. 171-187.

Hayek, F. von (1945): "The Use of Knowledge in Society", The American Economic Review, Vol. 35, Nr. 4, S. 519-530.

Hendershott, T. und Jones, C. (2005): "Island Goes Dark: Transparency, Fragmentation, and Regulation", in: Review of Financial Studies, Vol. 18, Nr. 3, S. 743-793.

Hengelbrock, J. und Theissen, E. (2009): "Fourteen at One Blow: The Market Entry of Turquoise", SSRN Working Paper.

http://papers.ssrn.com/sol3/papers.cfm?abstract_id=1570646 (Zugriff: 31.01.2013).

Holmström, B. und Myerson, R. (1983): "Efficient and Durable Decision Rules with Incomplete Information", in: Econometrica, Vol. 51, Nr. 6, S. 1799-1819.

Inderst, R. (2009): "Retail Finance: Thoughts on Reshaping Regulation and Consumer Protection after the Financial Crisis", in: European Business Organization Law Review, Vol. 10, S. 455-464.

Inderst, R. und Ottaviani, M. (2009): "Misselling through Agents", in: The American Economic Review, Vol. 99, Nr. 3, S. 883-908.

Jensen, M. (1978): "Some anomalous evidence regarding market efficiency", in: The Journal of Financial Economics, Vol. 6, Nr. 2-3, S. 95-101.

Klöhn, L. (2009): “Preventing Excessive Retail Investor Trading under MiFID: A Behavioural Law and Economics Perspective", in: European Business Organization Law Review, Vol. 10, Nr. 3, S. 437-454.

Lambert, R., Leuz, C. und Verrecchia, R. (2012): “Information Asymmetry, Information Precision, and the Cost of Capital", Review of Finance, Vol. 16, Nr. 1, S. 1-29.

Lee, C. (1993): "Market Integration and Price Execution for NYSE-Listed Securities", in: The Journal of Finance, Vol. 48, Nr. 3, S. 1009-1038.

Leuz, C. und Wysocki, P. (2008): "Economic Consequences of Financial Reporting and Disclosure Regulation: A Review and Suggestions for Future Research", SSRN Working Paper.

http://papers.ssrn.com/sol3/papers.cfm?abstract_id=1105398 (Zugriff: 06.02.2012).

Levine, R. (1997): "Financial Development and Economic Growth: Views and Agenda", in: Journal of Economic Literature, Vol. 35, S. 688-726.

Levitt, A. (1999): "Best Execution: Promise of Integrity, Guardian of Competition", Speech by SEC Chairman, Securities Industry Association, Boca Raton, Florida 4. November 1999.

http://www.sec.gov/news/speech/speecharchive/1999/spch315.htm (Zugriff: 29.12.12)

London Economics (2002): "Quantification of the Macro-Economic Impact of Integration of EU Financial Markets", Final Report to the European Commission - Directorate-General for the Internal Market. 
http://ec.europa.eu/internal_market/securities/docs/studies/summary-londonecon_en.pdf (Zugriff: 01.12.2012).

Madhavan, A., Porter, D. und Weaver, D. (2005): "Should Securities Markets be Transparent", in: Journal of Financial Markets, Vol. 8, S. 266-288.

Moher, D., Liberati, A., Tetzlaff, J. und Altman, D. (2009): "Preferred Reporting Items for Systematic Reviews and Meta-Analyses: The PRISMA Statement", in: Public Library of Science (PLoS) Medicine, Vol. 6, Nr. 7, S. 1-6.

Neal, L. und Davis, L. (2005): "The evolution of the rules and regulations of the first emerging markets: the London, New York and Paris stock exchanges, 1792-1914", in: The Quarterly Review of Economics and Finance, Vol. 45, Nr. 2-3, S. 296-311.

Neumann, M. und Klein, M. (1982): "Probleme der Theorie effizienter Märkte und ihrer empirischen Überprüfung", in: Kredit und Kapital, Vol. 15, Nr. 2, S. 165-187.

Pacces, A. (2000): "Financial intermediation in the securities markets law and economics of conduct of business regulation", in: International Review of Law and Economics, Vol. 20, Nr. 4, S. 479-510.

Pagano, M. (1989): "Trading volume and asset liquidity", in: Quarterly Journal of Economics, Vol. 104, S. 25-74.

Petrella, G. (2010): “MiFID, Reg NMS and Competition Across Trading Venues in Europe and United States", in: Journal of Financial Regulation and Compliance, Vol. 18, Nr. 3, S. 257-271.

Porter, D. und Weaver, D. (1998): “Post-trade transparency on Nasdaq's National Market System", in: The Journal of Financial Economics, Vol. 50, Nr.2, S. 231-252.

Posner, R. (1975): "The Social Costs of Monopoly and Regulation", in: The Journal of Political Economy, Vol. 83, Nr. 4, S. 807-828.

Ramb, B.-T. (1987): “Grundlagen der Wirtschaftspolitik", R. Oldenbourg.

Ribeiro, R. (2010): "Market Dominance and Barriers to Competition in Financial Trading Venues", NET Institute Working Paper Nr. 08-35.

http://papers.ssrn.com/sol3/papers.cfm?abstract_id=1287443 (Zugriff: 31.01.13).

Riordan, R., Storkenmaier, A. und Wagener, M. (2011): "Do Multilateral Trading Facilities Contribute to Market Quality?", SSRN Working Paper.

http://papers.ssrn.com/sol3/papers.cfm?abstract_id=1852769 (Zugriff: 31.01.13).

Rößner, C. (2008): “Außerbörslicher Handel - Gut für den Kunden!” in: Böhlen, A. von, und Kann, J. (Hrsg.): MiFID-Kompendium - Praktischer Leitfaden für Finanzdienstleister, Springer, S. 265-280.

Sell, J. (2006): “Miffed with MiFID", in: The Economist, 16.11.2006.

http://www.economist.com/node/8131795 (Zugriff: 26.02.2013).

Shleifer, A. (2005): "Understanding Regulation", in: European Financial Management, Vol. 11, Nr. 4, S. 439-451.

Soltani, B., Mai, H., und Jerbi, M. (2011): "Transparency and Market Quality: An Analysis of the Effect of MiFID on Euronext (May 1, 2011). International Conference of the French Fi- 
$\begin{array}{lllll}\text { nance } & \text { Association } & \text { (AFFI), } & \text { May }\end{array}$ http://papers.ssrn.com/sol3/papers.cfm?abstract_id=1833605 (Zugriff: 31.01.13).

Spankowski, U., Wagener, M. und Burghof, H.-P. (2012): "The Role of Traditional Exchanges in Fragmented Markets", SSRN Working Paper.

http://papers.ssrn.com/sol3/papers.cfm?abstract_id=1980951 (Zugriff: 31.01.13).

Steil, B. und Perfumo, D. (2003): "The Economics of Soft Dollar Trading", Working Paper.

Steennot, R. (2008): "Information Requirements as a Tool to Protect Consumers Receiving Investment Services", Univ. of Ghent Financial Law Institute Working Paper Nr. 2008-14. http://papers.ssrn.com/sol3/papers.cfm?abstract_id=1306294 (Zugriff: 31.01.13).

Stigler, G. (1975): "The Citizen and the State", The University of Chicago Press.

Stoll, H. (2003): "Market microstructure", in: Constantinides, G. M., Harris, M. und Stulz, R. M. (Hrsg.): Handbook of the Economics of Finance, 1. Aufl., Vol. 1, Elsevier, S. 553-604.

Storkenmaier, A. und Wagener, M. (2011): “Do we need a European 'National Market System'? Competition, Arbitrage, and Suboptimal Executions", SSRN Working Paper. http://papers.ssrn.com/sol3/papers.cfm?abstract_id=1760778 (Zugriff: 30.12.13).

Stringham, E., Boettke, P. und Clark, J. (2008): "Are Regulations the Answer for Emerging Stock Markets? Evidence from the Czech Republic and Poland", in: The Quarterly Review of Economics and Finance, Vol. 48, Nr. 3, S. 541-566.

Tinic, S. und West, R. (1979): "Investing in Securities: an Efficient Markets Approach", Addison-Wesley.

Tobin, J. (1984): "On the efficiency of the Financial System", in: Llyods Bank Review, Vol. 153, S. 1-15.

U.S. Securities and Exchange Commission (2005): "Regulation NMS", Release Nr. 34-51808. 